\title{
Comparative structural analysis of Bru 1 region homeologs in Saccharum spontaneum and S. officinarum
}

Jisen Zhang ${ }^{1,2,3}$, Anupma Sharma ${ }^{4}$, Qingyi Yu ${ }^{1,4}$, Jianping Wang ${ }^{1,5}$, Leiting Li ${ }^{3,6}$, Lin Zhu ${ }^{3,7}$, Xingtan Zhang ${ }^{1}$, Youqiang Chen ${ }^{2}$ and Ray Ming ${ }^{1,3^{*}}$

\begin{abstract}
Background: Sugarcane is a major sugar and biofuel crop, but genomic research and molecular breeding have lagged behind other major crops due to the complexity of auto-allopolyploid genomes. Sugarcane cultivars are frequently aneuploid with chromosome number ranging from 100 to 130, consisting of 70-80 \% S. officinarum, 10-20 \% S. spontaneum, and $10 \%$ recombinants between these two species. Analysis of a genomic region in the progenitor autoploid genomes of sugarcane hybrid cultivars will reveal the nature and divergence of homologous chromosomes.

Results: To investigate the origin and evolution of haplotypes in the Bru1 genomic regions in sugarcane cultivars, we identified two BAC clones from S. spontaneum and four from S. officinarum and compared to seven haplotype sequences from sugarcane hybrid R570. The results clarified the origin of seven homologous haplotypes in R570, four haplotypes originated from S. officinarum, two from S. spontaneum and one recombinant.. Retrotransposon insertions and sequences variations among the homologous haplotypes sequence divergence ranged from $18.2 \%$ to $60.5 \%$ with an average of 33 . $7 \%$. Gene content and gene structure were relatively well conserved among the homologous haplotypes. Exon splitting occurred in haplotypes of the hybrid genome but not in its progenitor genomes. Tajima's $D$ analysis revealed that $S$. spontaneum hapotypes in the Brul genomic regions were under strong directional selection. Numerous inversions, deletions, insertions and translocations were found between haplotypes within each genome.

Conclusions: This is the first comparison among haplotypes of a modern sugarcane hybrid and its two progenitors Tajima's D results emphasized the crucial role of this fungal disease resistance gene for enhancing the fitness of this species and indicating that the brown rust resistance gene in R570 is from S. spontaneum. Species-specific InDel, sequences similarity and phylogenetic analysis of homologous genes can be used for identifying the origin of $S$. spontaneum and S. officinarum haplotype in Saccharum hybrids. Comparison of exon splitting among the homologous haplotypes suggested that the genome rearrangements in Saccharum hybrids after hybridization. The combined minimum difference at $19.5 \%$ among homologous chromosomes in S. officinarum would be sufficient for proper genome assembly of this autopolyploid genome. Retrotransposon insertions and sequences variations among the homologous haplotypes sequence divergence may allow sequencing and assembling the autopolyploid Saccharum genomes and the auto-allopolyploid hybrid genomes using whole genome shotgun sequencing.
\end{abstract}

Keywords: Genome evolution, Haplotypes, Homologous genes, Polyploidy, Saccharum

\footnotetext{
* Correspondence: rming@life.uiuc.edu; rming@life.illinois.edu

${ }^{1}$ FAFU and UIUC-SIB Joint Center for Genomics and Biotechnology, Fujian

Provincial Key Laboratory of Haixia Applied Plant Systems Biology, Haixia

Institute of Science and Technology, Fujian Agriculture and Forestry

University, Fuzhou 350002, Fujian Province, China

${ }^{3}$ Department of Plant Biology, University of Illinois at Urbana-Champaign,

Urbana, IL 61801, USA

Full list of author information is available at the end of the article
} 


\section{Background}

Sugarcane (Saccharum spp.) is an important economic crop not only owing to its contribution of approximately $75 \%$ of world's sugar production, but also because of its leading role in biofuel production. Modern sugarcane cultivars are mostly interspecific hybrids derived from crosses between $S$. officinarum $(2 \mathrm{n}=8 \mathrm{x}=80)$ and $S$. spontaneum $(2 \mathrm{n}=40-128)$. Sugarcane cultivars are frequently aneuploid with chromosome number ranging from 100 to 130, consisting of 70-80\% S. officinarum, 10-20\% S. spontaneum, and $10 \%$ recombinants between these two species [1], though the classical cytogenetic studies concluded that there were no chromosomal exchanges between $S$. officinarum and S. spontaneum after hybridization [2-4]. Molecular mapping of hybrid R570 further confirmed that interspecific chromosome exchanges occurred between the two progenitor genomes $[5,6]$. GISH analyses of sugarcane hybrids indicated that the proportion of complete $S$. spontaneum chromosomes ranged from $10 \%$ to $23 \%$, and recombinant chromosomes of the two parental species varied from $5 \%$ to $17 \%$ in the hybrids $[7,8]$. Typically, the $\mathrm{F}_{1}$ hybrids and $\mathrm{BC}_{1}$ progeny receive $2 \mathrm{n}$ gametes from female $S$. officinarum parent and $\mathrm{n}$ gametes from male $S$. spontaneum parent during the interspecific hybridization, a phenomenon known as female restitution $(2 \mathrm{n}+\mathrm{n}$ chromosome transmission) [9].

The genome of modern sugarcane cultivar represents one of the most complex genomes studied to date. The ploidy level and genome size can vary significantly among commercial cultivars and other related Saccharum species. For example, the genome size of hybrid R570 was estimated at approximately $10 \mathrm{~Gb}$ with a ploidy level of $12 \mathrm{x}$ $[10,11]$, while the genome size of $S$. officinarum ranged from 7.50 to $8.55 \mathrm{~Gb}$ and that of S. spontaneum varied between 3.36 to $12.64 \mathrm{~Gb}$ [12]. So far, no sugarcane reference genome has been generated yet due to the complexity of autopolyploid genomes.

Sugarcane brown rust, caused by Puccinia melanocephala H\&P Syd., has been a severe fungal disease impacting sugarcane production for many years. The genetic resistance of the brown rust was identified in sugarcane cultivar, R570, which was controlled by a single dose dominant gene, Bru1. The resistance gene was initially mapped at a location, $10 \mathrm{cM}$ away from a restricted fragment length polymorphism (RFLP) probe, CDSR29 [13]. This resistance gene provides wide resistance against diverse brown rust isolates collected in both Africa and America [14]. Fine-mapping and physical mapping have been used to map this major durable resistance gene in an interval of two flanking markers, which were only $0.28 \mathrm{cM}$ and $0.14 \mathrm{cM}$ away from the Bru1 gene, respectively [15-17]. A physical map covering the two flanking markers was constructed through screening the bacterial artificial chromosome (BAC) clones in the existing R570 BAC library [10] and a Bru1-enriched BAC library [17]. The resulting physical map included three BAC clones from the target genotype with two remaining gaps and $32 \mathrm{BAC}$ clones from homologous haplotypes. Sequencing the clones in the target region revealed an inserted segment containing the target gene Bru1 in the target haplotype contig with two gaps, and the whole segment was absent in homologous haplotype contigs [17]. Sequencing eight BAC clones, including two clones from the target haplotype and six other clones from homologous haplotypes, has revealed 14 annotated genes. The comparison of the eight BAC clones' gapless sequences showed an average sequence identity of $97.7 \%$ in the exons and $96.9 \%$ in introns among the haplotypes. Based on the phylogenetic analysis of selected genes and sequence similarity of the seven haplotypes, four haplotypes were predicted to be derived from $S$. officinarum, two from S. spontaneum, and one being recombinant [18].

Comparative analysis between a sugarcane hybrid and its progenitor species is an effective approach to study the origin of sugarcane hybrid haplotypes, which will provide insights into chromosomal rearrangements after polyploidization and hybridization. LA Purple (S. officinarum, $2 \mathrm{n}=80$ ) and SES208 (S. spontaneum, $2 \mathrm{n}=64$ ) are varieties of the progenitor Saccharum species of modern sugarcane cultivars. With the available BAC libraries of LA Purple and AP85-441 (Ming and Yu, unpublished data), we investigated the sequence divergence among sugarcane hybrid (R570), S. officinarum (LA Purple) and S. spontaneum (AP85-441) in the Brul genomic region. The objectives of this project were to: 1) clarify the origin of sugarcane hybrid haplotypes in the Brul genomic region; 2) assess the evolutionary relationships of haplotypes within and between Saccharum species and interspecific hybrids; 3) evaluate the extent of DNA sequence divergence within major Saccharum species based on sugarcane haplotype sequences; and 4) evaluate selective constraint in genomic region containing candidate Brul gene. The comparative genomic study will improve our understanding of genome recombination and evolutionary relationships of Saccharum hybrids and its progenitor Saccharum species after hybridization.

\section{Methods \\ BAC libraries}

LA Purple (S. officinarum, $2 \mathrm{n}=80$ ) and AP85-441(the haploid clone of SES208, $2 n=4 x=32$ ) derived from the anther culture of SES208 [19] representing two major Saccharum species were used for BAC library construction. Nuclei were isolated from the young leaf tissues of LA Purple and SES208 haploid following the method 
described by Ming et al. [20]. The high molecular weight DNA embedded in agarose was partially digested using HindIII. The fraction at approximately $100 \mathrm{~kb}$ was recovered and cloned into pSMART BAC vector (Lucigen, LA). The BAC library of LA Purple consists of 74,880 clones in 195 384-well plates with average insert size at $150 \mathrm{~kb}$, providing $1.5 \mathrm{x}$ coverage of the octoploid genome and $12 x$ coverage of the monoploid genome. The BAC library of AP85-441consists of 38,400 clones in 100 384well plates with average insert size at $120 \mathrm{~kb}$, providing $1.5 \mathrm{x}$ coverage of the haploid (tetraploid) genome and $6 \mathrm{x}$ coverage of the monoploid genome.

\section{BAC clone screening and sequencing}

BAC library screening was carried out as described by $\mathrm{Yu}$ et al. [21]. For library screening, two probes about 500 bp were designed respectively based on the DNA sequence of two genes identified in Bru1 region: gene 8 and 11b [18]. Among the annotated genes in the haplotypes of hybrid R570, gene 10 is homologous to barley rust resistance protein with three missing exons. Gene 8 and gene $11 \mathrm{~b}$, which surround gene 10 , are highly conserved among the published Saccharum hybrid haplotype sequences [18]. Two probes corresponding to gene 8 and 11b respectively were used to screen the BAC libraries of LA Purple and AP85-441 haploid genomes (primers showed in Additional file 1).

To identify different haplotypes, the positive clones screened from the BAC library were then amplified used same primers for probes preparation, cloned into the pGEM $^{\circ}$-T Easy Vector Systems (Promega, A1360) and sequenced from both ends of the PCR product. The BAC clones representing different haplotypes were selected. The insert sizes of the identified BAC clones were estimated by comparing to standard size markers (NEB, N3552S) using CHEF gel electrophoresis.

The BAC DNAs were isolated using phaseprepTM BAC DNA kit (Sigma-Aldrich, NA0100-1KT) and the sequencing libraries were prepared individually with unique barcode for each clone. The sequencing libraries were then pooled and sequenced using Roche 454 Genome Sequencer FLX platform at Keck Center at UIUC. The raw reads were assembled using Roche/454 Newbler Assembler with default settings (http://www.my454.com/).

\section{Repeat database compiling and repeat masking}

To mask the repeats from the sugarcane BAC clone sequences for annotation, an in-house repeat database was compiled by assembling public available repeat databases and de novo assembling of sugarcane repetitive sequences.

To assemble the publicly available repeat databases, we downloaded TIGR plant repeat database (ftp://ftp.plant biology.msu.edu/pub/data/TIGR_Plant_Repeats/) [22], the
MIPS Repeat Element Database (mips-REdat) (ftp://ftpmi ps.helmholtz-muenchen.de/plants/REdat/) [23], Repbase (http://www.girinst.org/) [24] and the P-MITE database (without TSD for monocots from http://pmite.hzau.edu.cn/download/) [25]. Moreover, we also parsed 3470 GenBank sugarcane sequence accessions for features annotated as 'mobile_element', 'LTR', or 'repeat_region'. The unique repeats were extracted from each downloaded repeat database by removing the redundant repeats that have more than $95 \%$ sequence identity over $95 \%$ of the sequence length to other repeats. Some unique repeats were annotated in RepeatMasker format (id\#class/subclass) based on the repeat codes in their headers, if available, otherwise by comparing to the Repbase repeats using the RepeatClassifier script of the RepeatModeler package.

To de-novo identify the sugarcane repeats, available sugarcane sequences were parsed, including the sequences of the 96 sugarcane BAC clones (66 in-house BAC clones from LA Purple and AP85-441 libraries and 38 publicly available BAC clones from sugarcane hybrids (GenBank accessions AM403006-7, FJ348715-33, GU080318-23, GU207345-46, FN431661, FN431663-69, and HQ116788). The protein sequences of $A$. thaliana [26], B. distachyon [27], O. sativa [28], S. italica [29], Z. mays [30], and S. bicolor [31] were downloaded from Phytozome (http://www.phyto zome.net/) and combined to generate an in-house plant protein database. An in-house TE protein library was also compiled from TE protein libraries available with Maker [32], GypsyDB-2.0 [33], Transposon PSI (http://transposonp si.sourceforge.net/), and RepeatMasker [34] softwares. The de novo repeats were then predicted in the sugarcane BACs using the TEdenovo pipeline consisting of REPET package v2.2 [35] and using RepeatModeler-1.0.7 [36]. Gene fragments in the de-novo predicted repeats were identified based on their sequence similarity to plant proteins only but not TE proteins (E-value less than 0.1 using blastx) and were then $\mathrm{N}$-masked. The masked repeat sequences were split on Ns and resulting sequences classified using RepeatClassifier script of the RepeatModeler package based on similarity to known repeat proteins from TREP and RepeatMasker databases. Unclassified repeats were considered as repeats if these had more than 40 matches to the 104 sugarcane BACs at E-value less than $1 \mathrm{E}-20$ using blastn. Finally, the repeat database was made non-redundant using cd-hit $[37,38]$ with $95 \%$ identity and $95 \%$ coverage threshold. The final nonredundant de-novo sugarcane repeat database contained 845 repeats (representing 2, 605,348 nt) classified into 8 groups including 614 LTR retrotransposons, 167 transposons, 36 LINEs, 12 Helitrons, 8 Unknown, 6 SINEs, 1 simple repeat, and 1 satellite repeat. Sugarcane MITEs were predicted using MITEhunter [39] with default parameters.

The final in-house repeat database was then compiled by combining the unified and annotated public repeat 
database and the de novo identified sugarcane repeats. The repeat content of sugarcane BAC clone sequences in this study was determined by masking the BAC clone sequences using RepeatMasker against this compiled inhouse repeat database.

\section{Identification of transposable elements (TE) domains and estimation of TE insertion times}

To identify TE associated domains in the BAC clone sequences, rpsBLAST was used to search the BACs clone sequences against the conserved domain database (CDD) [40]. Overlapping TE domains aligned in the same orientation on the BAC clone sequences were fused as one TE domain and annotated based on the best domain hit in the CDD database. The LTR retrotransposons were identified in the sugarcane BACs based on the presence of TE domains. The two ends 5' and 3' LTRs were defined based on the sequence identity and the presence of target site duplications (TSD). The insertion time of full length LTR retrotransposons was calculated using the approach as described by San Mignel et al. [41]. The full length LTRs were aligned by MUSCLE [42] and the number of nucleotide substitutions per site $(k)$ between the $5^{\prime}$ and $3^{\prime}$ ends of LTRs was calculated using the Kimura 2-parameter model implemented in MEGA6 [43]. The $k$ values were converted to divergence time using the rate of $1.3 \mathrm{E}-8$ [44].

\section{Gene annotation}

The repeat-masked sequences were aligned against sugarcane expressed sequence tags (ESTs) comprised of 283,332 ESTs from GenBank, the unigene set of our inhouse sugarcane RNAseq data, and the sorghum gene models (Sorbi1_GeneModels_AllModels_20080319_nt.fas ta at http://genome.jgi-psf.org/Sorbi1/Sorbi1.download.ft p.html) using tblastx. The gene structures were further predicted using the online tool GeneSeqer (http://www. plantgdb.org/cgi-bin/GeneSeqer/index.cgi), and the ambiguities were checked and manually corrected according to the alignment of the sequences to sugarcane transcripts and sorghum gene models. The annotated genes from the sequences were compared to 52 corresponding genes in seven haplotypes from hybrid R570 (GenBank accessions: FN431661-FN431668) and 10 corresponding genes from a sorghum BAC (GenBank accessions: FN431669) [18].

To estimate the expression level of annotated genes in different tissues of LA Purple and AP85-441, we aligned RNAseq data of 42 million pair-end reads 20 million single-end reads from various tissues of LA Purple and AP85-441 respectively against the predicted cDNA sequences of annotated genes using Novoalign with default settings (http://www.novocraft.com/main/index.php). The number of aligned reads for each target gene were counted using Tablet [45]. The gene expression levels were calculated as fragments per kilobase of exon per million mapped fragments (RPKM) [46].

\section{Sequence divergence analysis}

Protein sequences of gene pairs were aligned with ClustalW 2.0 [47] The alignments were converted to codon alignment with PAL2NAL [48]. The substitution rates of synonymous (Ks) and non-synonymous (Ka) were estimated based on the YN method [49] using KaKs_Calculator 2.0 [50].

$\mathrm{Ka} / \mathrm{Ks}$ value differential significance analyses were performed using fisher exact test as implemented in KaKs_Calculator 2.0 [49]. The null hypotheses in fisher exact test is numbers of synonymous substitutions (Sd)/ number of synonymous sites $(S)=$ number of nonsysnonymous substitutions $(\mathrm{Nd}) /$ number of nonsysnonymous sites $(\mathrm{N})$, also means neutral mutation. Reject the null hypothesis if $\mathrm{Sd} / \mathrm{S}$ is significantly greater (negative selection) or smaller (positive selection) than $\mathrm{Nd} / \mathrm{N}$, as indicated by $\mathrm{P}$-value $<0.05$, and extremely significant if $P$-value $<0.01$. On the other hand, significance analyses for different groups of $\mathrm{Ka} / \mathrm{Ks}$ values was performed using Duncan's test with significance level of 0.05, which was implemented in agricolae package of $\mathrm{R}$ programming language [51]. A custom Perl script was used for SNP discovery based on pairwise sequence alignments (https:// github.com/lileiting/Pileup2singledose/tree/master/dnp).

Furthermore, we applied DnaSP 4.0 [49] to perform sliding window analysis for nucleotide diversity, Tajima's $\mathrm{D}$ and $\mathrm{Fu}$ and Li's D test with $1 \mathrm{kbp}$ window size and 100 bp step length.

\section{BAC sequence visualization and comparison}

The schematic of exons, conserved TE domains and repeats in sugarcane BAC sequences was generated using EasyFig [52]. Large-scale alignments between homologous BACs were performed using BLASTZ [53]. The BAC sequence comparison was performed using the Artemis Comparison Tool [54] and a genome alignment tool Mauve with default settings (http://gel.ahabs.wisc.edu/mauve/) [55].

\section{Results}

Screening and sequencing $S$. officinarum and $S$. spontaneum BAC clones containing Bru1 genomic region Nine positive clones from LA Purple and five from AP85441 were identified using two probes designed from genes 8 and 11 of the Brul genomic region. Among them, five from LA Purple and three from AP85-441 were confirmed by PCR. To distinguish the haplotypes and avoid sequencing the duplicated haplotypes, PCR fragments of gene 8 were cloned and sequenced, which confirmed four clones from LA Purple (So-57E04, So-96B11, So-99P01 and So- 
146H19) and two clones from AP85-441 BAC libraries (Ss-75D04 and Ss-23 K06) containing different homologous haplotypes. The insert sizes of six BAC clones ranged from $80 \mathrm{~kb}$ to $130 \mathrm{~Kb}$. These six clones were subjected to complete sequencing. The cleaned reads from each clone were assembled and yielded a total length of $559 \mathrm{~kb}$ for the six clones with average GC contents ranging from $44.2 \%$ to $46.8 \%$ (Table 1). The sequences of the six clones were deposited in Genbank (accession numbers: KP063111- KP063116).

Fifty-two genes were annotated from the sequences of the six BAC clones (Table 1 and Additional file 2). The average gene density was 1 gene/11.2 kb in LA Purple and 1 gene/9.5 kb in AP85-441 homologous BAC clone sequences. The total coding regions of predicted genes account for $35 \%$ and $38 \%$ of the sequences from LA Purple and AP85-441, respectively.

\section{Sequence comparison between homologous haplotypes} Comparative analysis was performed between the homologous BAC sequences of LA Purple, So-57E04, So96B11, So-99P01 and So-146H19. Pair-wise sequence alignments revealed insertions and deletions in all six pairs (Additional file 3: Figure S1.1-1.6 and Additional file 4). The large InDels were observed mainly in intergenic regions between gene 5 and gene 6 , gene 6 and gene 7 , and within the genic regions of gene 4 , gene 5 , gene10 and gene 11a (Table 2). The alignment gaps appeared in all pairs and were not equally distributed, ranging from $18.2 \%$ to $60.5 \%$ (33.7 \% in average) of the aligned regions. The four haplotypes shared identities of approximately $96.8 \%$ in average ranged from $95.44 \%$ to $98.39 \%$, and an average of $1.8 \%$ SNPs difference with a range of $1.66 \%$ to $2.48 \%$ on the gapless alignments of the corresponding regions (Table 3 ). The average divergence among the haplotypes in S. officinarum is $3.2 \%$. Besides, inversions were clearly observed in alignments of all pairs excepting the So-57E04/So-96B11 (Additional file 3: Figure S1.6).

Between the homologous sequences of $S$. spontaneum BAC clones, Ss-75D04 and Ss-23 K06, 72,274 bp (from 302 bp to 72,576 bp) from Ss-75D04 were aligned with 88,625 bp (from 287 bp to 88,912 bp) from Ss-23 K06, showing a 16,351 bp (18.4 \%) expansion in Ss-23 K06. These two BACs shared an average sequence identity at $98.47 \%$ and had $1.30 \%$ of SNPs on the gapless comparison. InDels were observed between these two BACs (Additional file 3: Figure S1.7). Two large transposable elements, belonging to DNA/MULE-MuDR and LTR/ Copia families, were found at regions 19,051-23,834 and 62,361-71,846 in Ss-23 k06, respectively (Fig. 1 and Additional file 5). In the genic regions, a 4,818 bp insertion in intron 2 of gene 4 and a 566 bp insertion in intron 4 of gene 5 were found on the Ss-23 K06 (Fig. 1 and Additional file 6: Figure S 4.5), presenting a $14.3 \%$ $(5,374 / 37,661$, length of insertions/length of 11 genes) expansions in genic region of the BAC sequences. These results indicated that the expansion on haplotype of Ss$23 \mathrm{~K} 06$ was originated from transposable element insertions in both genic and intergenic regions.

Sequence comparison between haplotypes of $S$. spontaneum and S. officinarum showed large InDels in intergenic regions between two pairs of genes: genes 5 and 6, genes 6 and 7, and within the genic regions of two pairs of genes: genes 4 and 5, genes 10 and 11a. In addition, large segmental insertions were observed in intergenic regions between genes 9 and 11b in all the haplotypes of S. officinarum, which can be used as a $S$. officinarum-specific marker to distinguish the origin of haplotypes in hybrids. Unevenly distributed alignment gaps existed in all eight pairs of BACs ranging from $18.2 \%$ to $60.5 \%$ of the aligned sequence. Inversions were found in the corresponding regions between genes 5 and 6 of paired haplotypes of Ss-23 K06/So-146H19 and Ss-75D04/ So-146H19. Further sequence analysis has shown

Table 1 Summary of the sequence length, GC content, transposable element content, and gene number

\begin{tabular}{|c|c|c|c|c|c|c|c|c|}
\hline \multirow[t]{2}{*}{ Species } & \multirow[t]{2}{*}{ NO } & \multirow[t]{2}{*}{ BAC ID } & \multirow[t]{2}{*}{ Length (Kb) } & \multirow{2}{*}{$\begin{array}{l}\text { GC } \\
\text { content }\end{array}$} & \multicolumn{3}{|c|}{ Transposable elements } & \multirow{2}{*}{$\begin{array}{l}\text { Gene } \\
\text { numbe }\end{array}$} \\
\hline & & & & & LTR & Non-LTR & Transposons & \\
\hline \multirow{4}{*}{$\begin{array}{l}\text { S. officinarum } \\
\text { (LA Purple) }\end{array}$} & 1 & $146 \mathrm{H} 19$ & 77.5 & $44.7 \%$ & $16.91 \%$ & $1.92 \%$ & $29.47 \%$ & 8 \\
\hline & 2 & 99P01 & 74.4 & $44.2 \%$ & $35.94 \%$ & $2.46 \%$ & $28.97 \%$ & 7 \\
\hline & 3 & $96 \mathrm{~B} 11$ & 101.3 & $46.8 \%$ & $35.12 \%$ & $0.05 \%$ & $24.55 \%$ & 8 \\
\hline & 4 & 57E04 & 95.3 & $45.0 \%$ & $34.90 \%$ & $3.57 \%$ & $16.42 \%$ & 8 \\
\hline \multirow{2}{*}{$\begin{array}{l}\text { S. spontaneum } \\
\text { (AP85-441) }\end{array}$} & 5 & 75D04 & 72.0 & $44.7 \%$ & $11.13 \%$ & $6.57 \%$ & $36.23 \%$ & 10 \\
\hline & 6 & $23 \mathrm{KO6}$ & 127.7 & $45.3 \%$ & $26.66 \%$ & $4.70 \%$ & $25.31 \%$ & 11 \\
\hline Average & & & 91.5 & $45.2 \%$ & $27.48 \%$ & $3.20 \%$ & $26.16 \%$ & 8.7 \\
\hline Average (LA) & & & & & $31.19 \%$ & $1.94 \%$ & $24.36 \%$ & \\
\hline Average (SES) & & & & & $21.02 \%$ & $6.14 \%$ & $29.27 \%$ & \\
\hline Total & & & 549 & - & & & & 52 \\
\hline
\end{tabular}


Table 2 Repeat content in the haplotype sequences of LA Purple (S.officinarum), AP85-441 (S. spontaneum), and the hybrid cultivar, R570

\begin{tabular}{|c|c|c|c|c|c|c|c|c|c|}
\hline Species & \multicolumn{3}{|c|}{ Saccharum spontaneum } & \multicolumn{3}{|c|}{ Saccharum officinarum } & \multicolumn{3}{|c|}{ Saccharum hybrid R570 } \\
\hline Number of BACs & \multicolumn{3}{|c|}{2} & \multicolumn{3}{|c|}{4} & \multicolumn{3}{|c|}{8} \\
\hline \multirow[t]{2}{*}{ Total length of BAC (bp) } & \multirow{2}{*}{\multicolumn{3}{|c|}{$\begin{array}{c}200387 \\
\text { (198937 exclusing Ns) }\end{array}$}} & \multirow{2}{*}{\multicolumn{3}{|c|}{$\begin{array}{c}348447 \\
(346526 \text { excluding Ns) }\end{array}$}} & \multirow{2}{*}{\multicolumn{3}{|c|}{$\begin{array}{c}960468 \\
\text { (960468 excluding Ns) }\end{array}$}} \\
\hline & & & & & & & & & \\
\hline Type of repetitive elements & Number & Length & $\%$ & Number & Length & $\%$ & Number & Length & $\%$ \\
\hline \multirow{3}{*}{$\begin{array}{c}\text { Retroelements } \\
\text { SINEs: } \\
\text { LINEs: }\end{array}$} & 52 & 52906 & 26.40 & 50 & 115439 & 33.13 & 207 & 422842 & 44.02 \\
\hline & 2 & 1211 & 0.60 & 2 & 528 & 0.15 & 6 & 3214 & 0.33 \\
\hline & 16 & 9575 & 4.78 & 10 & 6238 & 1.79 & 54 & 70279 & 7.32 \\
\hline L1 & 11 & 6840 & 3.41 & 9 & 5305 & 1.52 & 49 & 65315 & 6.80 \\
\hline RTE-BovB & 5 & 2735 & 1.36 & 1 & 933 & 0.27 & 4 & 2654 & 0.28 \\
\hline Unknown/Other & 0 & 0 & 0.00 & 0 & 0 & 0.00 & 1 & 2310 & 0.24 \\
\hline LTR elements: & 34 & 42120 & 21.02 & 38 & 108673 & 31.19 & 147 & 349349 & 36.37 \\
\hline Copia & 17 & 32535 & 16.24 & 17 & 65941 & 18.92 & 36 & 111526 & 11.61 \\
\hline Gypsy & 11 & 8765 & 4.37 & 19 & 42012 & 12.06 & 66 & 202830 & 21.12 \\
\hline Unknown & 6 & 820 & 0.41 & 2 & 720 & 0.21 & 45 & 34993 & 3.64 \\
\hline DNA transposons & 60 & 58662 & 29.27 & 78 & 84896 & 24.36 & 222 & 230236 & 23.97 \\
\hline Tc1-Mariner & 12 & 17507 & 8.74 & 11 & 11727 & 3.37 & 31 & 38721 & 4.03 \\
\hline CMC-EnSpm (САCTA) & 11 & 6335 & 3.16 & 7 & 14953 & 4.29 & 108 & 119999 & 12.49 \\
\hline MULE & 8 & 3728 & 1.86 & 4 & 2518 & 0.72 & 7 & 3554 & 0.37 \\
\hline PIF-Harbinger & 29 & 31092 & 15.52 & 56 & 55698 & 15.98 & 70 & 62416 & 6.50 \\
\hline hAT-Ac & 0 & 0 & 0.00 & 0 & 0 & 0.00 & 5 & 1710 & 0.17 \\
\hline IS & 0 & 0 & 0.00 & 0 & 0 & 0.00 & 1 & 3836 & 0.40 \\
\hline Unclassified & 0 & 0 & 0.00 & 3 & 5395 & 1.55 & 6 & 14085 & 1.47 \\
\hline Total interspersed repeats & 112 & 111568 & 55.68 & 131 & 205730 & 59.04 & 435 & 667163 & 69.46 \\
\hline Low complexity: & 4 & 168 & 0.08 & 8 & 430 & 0.12 & 8 & 429 & 0.04 \\
\hline Satellite & 0 & 0 & 0.00 & 0 & 0 & 0.00 & 1 & 95 & 0.01 \\
\hline Simple_repeat & 44 & 1618 & 0.81 & 54 & 2559 & 0.73 & 126 & 5515 & 0.57 \\
\hline
\end{tabular}

* Remarkable differences in repeat content are marked by yellow (low) and red (high) highlights.

that the inverted segment of $48,541-51,370$ in So$146 \mathrm{H} 19$ might be originated from the duplication and inversion of the segment of 45,239-48,068 (Additional file 3: Figure S1.15). Based on the gapless alignments of the corresponding regions, the two haplotypes of $S$. spontaneum shared an average of $96.1 \%$ (ranging from $94.5 \%$ to $97.0 \%$ ) sequence identities and showed an average of $2.0 \%$ SNPs (ranging from $1.7 \%$ to $2.5 \%$ ) with the four haplotypes from S. officinarum (Table 3).

Identification of SNPs in the Bru1 homologous haplotypes In the Bru1 homologous haplotypes, pairwise sequences alignments were performed for discovering SNPs within and among Saccharum Species in the gapless regions (Table 4). 3501, 881 and 6389 SNPs corresponding to
$196,401 \mathrm{bp}, 67,783 \mathrm{bp}$ and $311,687 \mathrm{bp}$ of aligned sequences were identified within S. officinarum, S. spontaneum and between S.officinarum and S. spontaneum, respectively. The SNP densities were higher in between S. officinarum and S. spontaneum than within each of the two Saccharum species.

To evaluate the distributions of SNPs and the possibility of distinguishing the homologous haplotypes in Saccharum genomes, the SNP number of the fragment with sizes of $100 \mathrm{bp}, 250 \mathrm{bp}, 500 \mathrm{bp}$ and $1000 \mathrm{bp}$ were identified in the pairwise alignment in the Bru1 homologous haplotypes. The results revealed that $50.22 \%$ of 100 bp sequences pairs and $27.52 \%$ of SNPs of $250 \mathrm{bp}$ sequences pairs had no SNPs in S. spontaneum. S. spontaneum were observed to exhibit more identical sequences between the two Brul homo(eo)logous haplotypes (Table 4). 
Identification of species-specific haplotypes in R570

Segmental InDels between gene 10 and gene $11 \mathrm{~b}$ were presented in S. officinarum LA Purple and absent in $S$. spontaneum AP85-441. These InDels can be used to identify the species-specific haplotypes in Saccharum hybrid R570. Based on the large S. officinarum insertion fragment, the six haplotypes from hybrid R570 can be sorted into two groups, one group including BACs 142 J21, 135P16, 253G12 and CIR9020/12E03 with the insertion as in S. officinarum, and the other group including BACs 15 N23 and 197G04 without the insertion as in S. spontaneum. 53A11 was not grouped together with the other BACs because it does not have the corresponding homolog sequence (Table 5 and Fig. 1).

The sequence comparisons provided a reference for distinguishing the haplotype origin in Saccharum hybrids. The four haplotypes with the large inserted fragment from R570 shared higher sequence similarity (96.62 to $98.38 \%$ ) with $S$. officinarum than that with $S$. spontaneum (94.56 to $95.88 \%$ ) (Table 5). The sequence of BAC clone 53A11 missing the corresponding insertion also presented higher sequence similarity (96.41\%) with S. officinarum than that with S. spontaneum (95.67 \%). BACs 15 N23 and 197G04 shared $96.07 \%$ and $97.24 \%$ sequences identities with $S$. spontaneum haplotypes, and 96.61 and $93.82 \%$ with S. officinarum haplotypes, respectively (Table 6).

Furthermore, to verify the prediction by Garsmeur et al. [18], similar analyses with phylogenetic tree and haplotype networks were used to identify the origin of the Brul region in Saccharum hybrids with homologous sequences from two progenitor Saccharum Species as references. To be comparable, a similar synthetic representation as Garsmeur et al. [18] for the results is presented in Fig. 2. The maximum divergence between two alleles within a locus ranges from 2.34 to 9.61 MYRs. Sh15N23, CIR9020-12E03 and Ss-23 K06 contain two of the most divergent gene alleles. Based on the phylogenetic analysis of gene alleles, genes 6,8 , and $11 \mathrm{~b}$ were all grouped separately from $S$. spontaneum and S. officinarum haplotypes; gene 7 from two S. spontaneum and three out of four S.officinarum haplotypes (beside So57E04) were grouped. Therefore, the hybrid BACs 142 J21, 135P16, 253G12, and 53A11 should be originated from $S$. officinarum as shown by phylogenetic groups of genes 6, 7 and 8, while, hybrid BACs 15 N23 and 197G04 should be from S. spontaneum according to phylogenetic group of genes $6,7,8$ and $11 \mathrm{~b}$ (Fig. 2). In addition, in haplotype CIR9020-12E03, genes 6,7 , and 8 were grouped with the S. officinarum alleles, and gene 11a in the S. officinarum specific InDel region was presented, while, $11 \mathrm{~b}$ was grouped together with the $S$. spontaneum alleles. This result demonstrated that CIR9020-12E03 was a haplotype with a recombinant region between gene 11a and 11b. Our results confirmed the prediction by Garsmeur et al. that of the seven haplotypes from Saccharum hybrids, four were derived from S. officinarum, two from S. spontaneum and the remaining one was from recombinant.

\section{Gene arrangements and structures in homologous haplotypes}

The lack of genes 10 and 11a was observed in haplotypes of S. spontaneum (AP85-441) in comparison to haplotypes of S. officinarum (LA Purple) (Fig. 1 and Table 5). Similarly, genes 10 and 11a were only found in our in-house RNAseq database of S. officinarum, but not in S. spontaneum. Except these two genes, all the other genes remained the same order and orientation with conserved sizes and coding sequences in both species. Pseudogenes were found in alleles of both Saccharum species. In the two haplotypes from S. spontaneum (AP85-441), a premature stop codon caused by an insertion was found in the exon 11 of gene 5 . In $S$. officinarum, a premature stop codon was also observed in the exon 6 of gene 3 and exon 1 of gene 8 in the haplotype of So-96B11, which resulted two pseudogenes. Two large insertions, $21 \mathrm{~kb}$ and $17 \mathrm{~kb}$, were found in the introns of genes 10 in haplotypes of So-57E04 and So99P01, respectively (Additional file 6). 4 out of the 28 genes (gene 10 was not included) in two Saccharum species were identified to be pseudogenes (Table 5).

Comparing the genomic region in two species and hybrid R570, the genes in haplotypes of 15 N23 and 197G04 from R570 remained the same order, orientation and missed genes (10 and 11a) as presented in the haplotypes of S. spontaneum (AP85-441). The remaining five of six haplotypes from hybrid BACs showed the same order and orientation as in haplotypes of S. officinarum (LA Purple). All the sequence alignment and gene comparison indicated that the two haplotypes of 15 N23 and 197G04 are originated from S. spontaneum, validating prediction of Garsmeur et al. [18]. In hybrids, except gene 10, the coding regions of all the other genes could be translated into complete protein sequences [18].

Comparing homologous sequences between sugarcane and sorghum, gene 9, gene 10 and gene 11a were absent in sorghum. Only the first exon of gene 9 was retained in sorghum. Large InDels were found in the region between genes 8 and gene 11b between sugarcane and sorghum, which might indicate that the region between genes 8 and gene 11b was a hotspot of genome rearrangement in Saccharum.

The structure of each gene was analyzed. The gene size differences were mainly caused by the variations of intron length. Except for genes 1, 2 and 9, the other 10 unique genes exhibited distinct sizes of introns among the haploytypes (Additional file 6). Furthermore, LTR insertions were found in genes $4,10,11 \mathrm{a}$ and $11 \mathrm{~b}$, which caused the intron size variation in these genes. However, the intron variations occurred randomly among different haplotypes. By contrast, coding regions were conserved 
Table 3 Summary of gapless sequence comparison the haplotypes BACS among S. officinarum(LA Purple) and S. spontaneum(AP85-441)

\begin{tabular}{|c|c|c|c|c|c|c|}
\hline BAC name & BAC name & Ss-75D04 & So-57E04 & So-96B11 & So-99P01 & So-146H19 \\
\hline $\begin{array}{l}\text { (Length } \\
\text { bp) }\end{array}$ & Length (bp) & 71,995 & 95,342 & 101,291 & 74,354 & 77,460 \\
\hline Ss-23 K06 & $\begin{array}{l}\text { range of aligned } \\
\text { sequence }\end{array}$ & $(287-88918) /(302-72582)$ & $(39884-95090) /(17837-88219)$ & $(168-81137) /(8608-80258)$ & $(37335-85913) /(26-66382)$ & $(6628-78055) /(5672-77310)$ \\
\hline \multirow[t]{5}{*}{127,658} & Span of aligned BAC & $\begin{array}{l}68512(77.30 \%) / \\
68716(95.07 \%)\end{array}$ & $\begin{array}{l}33316(60.35 \%) / \\
33467(47.55 \%)\end{array}$ & $\begin{array}{l}51174(63.20 \%) / \\
51331(71.64 \%)\end{array}$ & $\begin{array}{l}29192(60.09 \%) / \\
29187(43.99 \%)\end{array}$ & $\begin{array}{l}49413(69.18 \%) / \\
49502(69.10 \%)\end{array}$ \\
\hline & Aligned sequence & 67783 & 32441 & 50240 & 28799 & 48249 \\
\hline & Average identity (\%) & 98.37 & 95.92 & 96.08 & 96.72 & 94.53 \\
\hline & SNP (\%) & $881(1.30 \%)$ & $620(1.91 \%)$ & $1035(2.06 \%)$ & $506(1.76 \%)$ & $1153(2.39 \%)$ \\
\hline & MNP(\%) & 0.43 & 2.17 & 1.86 & 1.52 & 3.08 \\
\hline Ss-75D04 & $\begin{array}{l}\text { range of aligned } \\
\text { sequence }\end{array}$ & & $(35029-72582) /(17837-83938)$ & $(302-65299) /(8743-80258)$ & $(32480-72726) /(26-74352)$ & $(6303-62979) /(5672-77310)$ \\
\hline \multirow[t]{5}{*}{71,995} & Span of aligned BAC & & $\begin{array}{l}29553(78.70 \%) / \\
29618(44.81 \%)\end{array}$ & $\begin{array}{l}49688(76.45 \%) / \\
49719(69.52 \%)\end{array}$ & $\begin{array}{l}29384(73.01 \%) / \\
29378(39.53 \%)\end{array}$ & $\begin{array}{l}46112(81.36 \%) / \\
46037(64.26 \%)\end{array}$ \\
\hline & Aligned sequence & & 28814 & 48973 & 28938 & 45233 \\
\hline & Average identity (\%) & & 95.80 & 96.48 & 97.00 & 96.36 \\
\hline & SNP (\%) & & $479(1.66 \%)$ & $984(2.01 \%)$ & $489(1.69 \%)$ & $1123(2.48 \%)$ \\
\hline & & & 2.54 & 1.51 & 1.31 & 1.16 \\
\hline So-57E04 & $\begin{array}{l}\text { range of aligned } \\
\text { sequence }\end{array}$ & & & $(9475-36844) /(39893-80175)$ & $(17837-95341) /(6441-67799)$ & $(10894-39851) /(28927-77310)$ \\
\hline \multirow[t]{5}{*}{95,342} & Span of aligned BAC & & & $\begin{array}{l}21851(79.84 \%) / \\
21895(54.35 \%)\end{array}$ & $\begin{array}{l}57223(73.83 \%) / \\
57303(93.39 \%)\end{array}$ & $\begin{array}{l}24491(84.58 \%) / \\
24304(50.23 \%)\end{array}$ \\
\hline & Aligned sequence & & & 21601 & 56769 & 23815 \\
\hline & Average identity (\%) & & & 95.99 & 97.28 & 95.44 \\
\hline & SNP (\%) & & & $377(1.75 \%)$ & $993(1.75 \%)$ & $473(1.99 \%)$ \\
\hline & MNP(\%) & & & 2.26 & 0.97 & 2.57 \\
\hline So-96B11 & $\begin{array}{l}\text { range of aligned } \\
\text { sequence }\end{array}$ & & & & $(48130-80175) /(38-27603)$ & $(14403-80175) /(5672-71165)$ \\
\hline \multirow[t]{5}{*}{101,291} & Span of aligned BAC & & & & $\begin{array}{l}23235(72.51 \%) / \\
23221(84.24 \%)\end{array}$ & $\begin{array}{l}47909(72.84 \%) / \\
47707(72.84 \%)\end{array}$ \\
\hline & Aligned sequence & & & & 23083 & 46867 \\
\hline & Average identity (\%) & & & & 98.39 & 96.57 \\
\hline & SNP (\%) & & & & $364(1.58 \%)$ & $853(1.82 \%)$ \\
\hline & MNP(\%) & & & & 0.03 & 1.61 \\
\hline
\end{tabular}


Table 3 Summary of gapless sequence comparison the haplotypes BACs among S. officinarum(LA Purple) and S. spontaneum(AP85-441) (Continued)

So-99P01 $\begin{aligned} & \text { range of aligned } \\ & \text { sequence }\end{aligned}$

\section{sequence}

74,354 Span of aligned BAC

(24-30055)/(34688-77310)

$24568(81.81 \%) /$

$24554(57.61 \%)$

Aligned sequence

24266

Average identity (\%)

96.98

SNP (\%)

$441(1.82 \%)$

$\mathrm{MNP}(\%)$

1.20

Notes: A summary of gapless sequence comparison the haplotypes BACs among S.officinarum(LA Purple), S.spontaneum(AP85-441) and Saccarhum cultivar R570 was presented in table S. 4. SNP: single nucleotide polymorphism; MNP: Multiple nucleotide Polymorphisms 


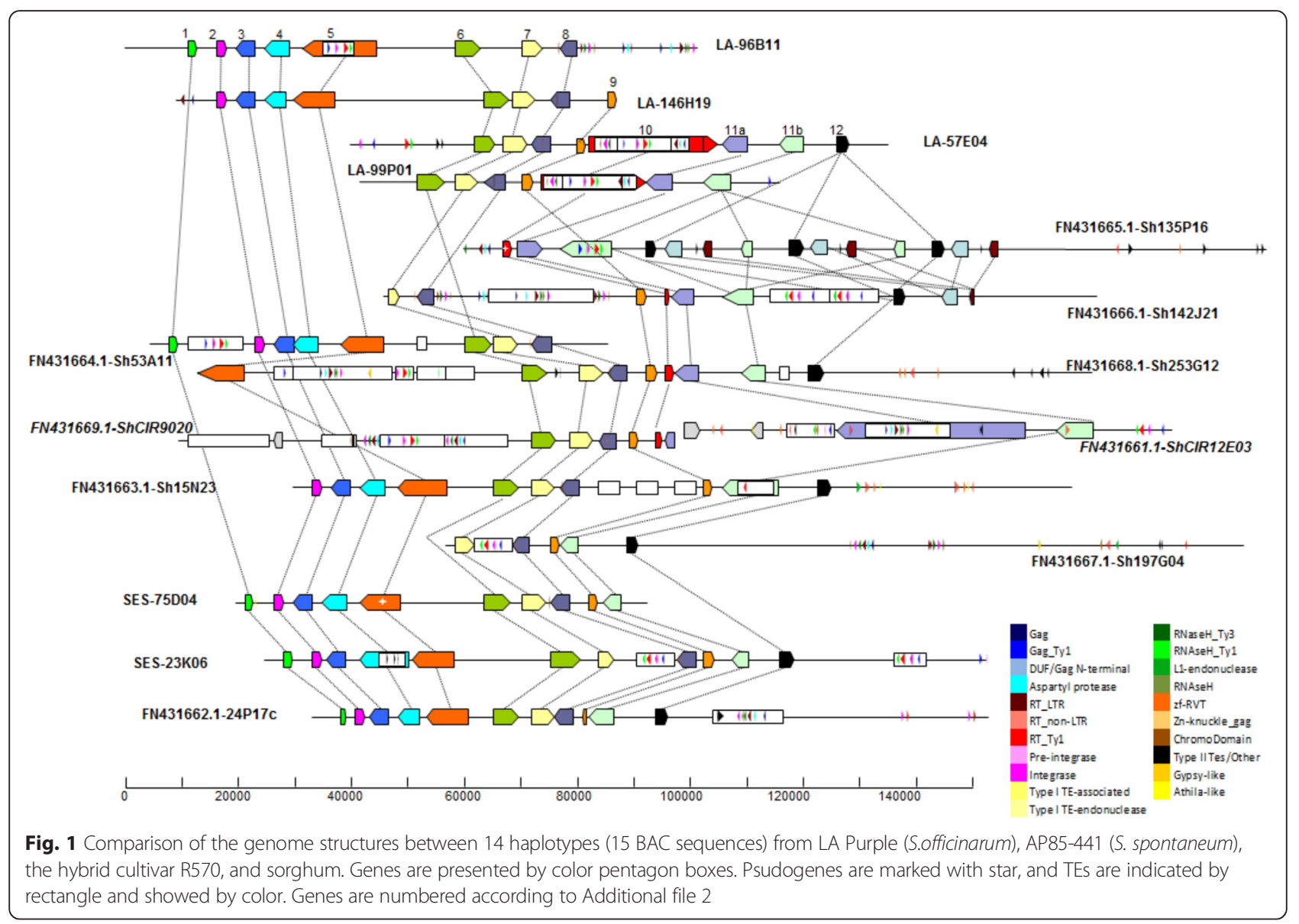

among haplotypes. Of the 13 unique genes, exon splitting occurred in genes 1 and 8 of haplotype ShIV (FN31664.1) and ShV (FN31666.1) from the hybrid, respectively. However, these genes preserved their coding and putative amino acid sequences among all the alleles in the two progenitor species.

\section{Selective constraints on homologous genes between the haplotypes of S. officinarum, S. spontaneum and Saccharum hybrid}

The $\mathrm{Ka} / \mathrm{Ks}$ ratio of 12 pairs of genes was compared between the haplotypes of the two Saccharum species and
Saccharum hybrids to estimate the selective constraints for the homologs (Additional file 7). In comparison, no significant difference of $\mathrm{Ka} / \mathrm{Ks}$ ratios were observed in the homologous genes among Saccharum species (Additional file 8). Among the 13 unique genes, 451 gene pairs from Saccharum species, Saccharum hybrids and Sorghum were used for $\mathrm{Ka} / \mathrm{Ks}$ analysis. The $\mathrm{Ka} / \mathrm{Ks}$ ratios of 428 gene pairs $(94.5 \%$ ) were less than 1 while the $\mathrm{Ka} / \mathrm{Ks}$ ratio of the rest 23 gene pairs was above 1 . These results suggested that the majority of homologous alleles were under purifying selection. Gene pairs of $11 \mathrm{~b}$ in S. spontaneum haplotypes were found to under stronger purifying selection

Table 4 Pairwise SNPs distributions in Saccharum species

\begin{tabular}{|c|c|c|c|c|c|c|c|c|c|c|c|}
\hline & \multirow{2}{*}{$\begin{array}{l}\text { Aligned } \\
\text { sequence(bp) }\end{array}$} & \multirow{2}{*}{$\begin{array}{l}\text { SNP } \\
\text { number }\end{array}$} & \multirow[t]{2}{*}{ SNP \% } & \multicolumn{2}{|c|}{$100 \mathrm{nt}$} & \multicolumn{2}{|c|}{$250 \mathrm{nt}$} & \multicolumn{2}{|c|}{$500 \mathrm{nt}$} & \multicolumn{2}{|c|}{$1000 \mathrm{nt}$} \\
\hline & & & & $\overline{S D}$ & 0 SNPs (\%) & $\mathrm{SD}$ & 0 SNPs (\%) & SD & 0 SNPs(\%) & $\mathrm{SD}$ & 0 SNPs(\%) \\
\hline So & 196401 & 3501 & 1.78 & 1.73 & 31.35 & 3.57 & 11.26 & 6.52 & 2.77 & 11.77 & 0.40 \\
\hline Ss & 67783 & 881 & 1.30 & 1.34 & 50.22 & 2.94 & 27.52 & 5.51 & 22.30 & 9.78 & 17.39 \\
\hline $\mathrm{Ss} / \mathrm{SO}$ & 311687 & 6389 & 2.05 & 1.76 & 18.66 & 3.54 & 3.31 & 6.27 & 0.49 & 11.32 & 0.00 \\
\hline
\end{tabular}

Notes

The SNPs discovery was based on pairwise gapless sequence comparisons

0 SNPs (\%): The percentage of fragments for specific sizes (100 nt, $250 \mathrm{nt}, 500 \mathrm{nt}$ and $1000 \mathrm{nt}$ ) that contained no SNP

SD: The standard variation of SNP numbers among the specific sizes of fragments

So: S. officinarum, Ss: S. spontaneum 
Table 5 The feature of syntenic genes on Saccharum and sorghum bacterial artificial chromosome (BAC) clones

\begin{tabular}{|c|c|c|c|c|c|c|c|c|c|c|c|c|c|c|c|}
\hline & & \multicolumn{2}{|c|}{ S. spontaneum } & \multicolumn{4}{|c|}{ S. officinarum } & \multicolumn{2}{|c|}{$\begin{array}{l}\text { Saccharum hybrid-S. } \\
\text { spontaneum }\end{array}$} & \multicolumn{4}{|c|}{ Saccharum hybrid-S. officinarum } & \multirow{3}{*}{$\begin{array}{l}\text { Saccharum hybrid } \\
\text { recombination } \\
\text { CIR9O20/12E03 }\end{array}$} & \multirow{3}{*}{$\begin{array}{l}\text { Sorghum } \\
\text { 24P17 } \\
\text { Sorghum }\end{array}$} \\
\hline & & Ss-75D04 & Ss-23 K06 & So-99P01 & So-57E04 & So-96B11 & So-146H19 & $15 \mathrm{~N} 23$ & $197 G 04$ & $142 \mathrm{~J} 21$ & $135 \mathrm{P} 16$ & $253 \mathrm{G} 12$ & $53 \mathrm{~A} 11$ & & \\
\hline & & & & & & & & & & & & & & & \\
\hline \multirow[t]{4}{*}{ Gene1 } & DNA & 810 & 813 & - & - & 813 & - & - & - & - & - & - & 996 & - & 561 \\
\hline & Exons & 1 & 1 & - & - & 1 & - & - & - & - & - & - & 2 & - & 1 \\
\hline & CDNA & 810 & 813 & - & - & 813 & - & - & - & - & - & - & 741 & - & 561 \\
\hline & Amino acids & 269 & 270 & - & - & 270 & - & - & - & - & - & - & 246 & - & 186 \\
\hline \multirow[t]{4}{*}{ Gene2 } & DNA & 1180 & 1195 & - & - & 1195 & 1195 & 1192 & - & - & - & - & 1195 & - & 1192 \\
\hline & Exons & 2 & 2 & - & - & 2 & 2 & 2 & - & - & - & - & 2 & - & 2 \\
\hline & cDNA & 1086 & 1101 & - & - & 1101 & 1101 & 1098 & - & - & - & - & 1101 & - & 1098 \\
\hline & Amino acids & 361 & 366 & - & - & 366 & 366 & 365 & - & - & - & - & 366 & - & 365 \\
\hline \multirow[t]{4}{*}{ Gene3 } & DNA & 3216 & 3214 & - & - & $3223^{a}$ & 3356 & 3449 & - & - & - & - & 3203 & - & 3193 \\
\hline & Exons & 7 & 7 & - & - & 7 & 7 & 7 & - & - & - & - & 7 & - & 7 \\
\hline & CDNA & 948 & 948 & - & - & $953^{a}$ & 948 & 975 & - & - & - & - & 948 & - & 975 \\
\hline & Amino acids & 315 & 315 & - & - & $-^{a}$ & 315 & 324 & - & - & - & - & 315 & - & 324 \\
\hline \multirow[t]{4}{*}{ Gene4 } & DNA & 3894 & 8712 & - & - & 3910 & 3516 & 3888 & - & - & - & - & 3515 & - & 3330 \\
\hline & Exons & 10 & 10 & - & - & 10 & 10 & 10 & - & - & - & - & 10 & - & 10 \\
\hline & CDNA & 1002 & 1002 & - & - & 1002 & 993 & 960 & - & - & - & - & 1002 & - & 1032 \\
\hline & Amino acids & 333 & 333 & - & - & 333 & 330 & 319 & - & - & - & - & 333 & - & 343 \\
\hline \multirow[t]{4}{*}{ Gene5 } & DNA & $6972^{\mathrm{a}}$ & $6954^{\mathrm{a}}$ & - & - & 12770 & 7312 & 7912 & - & - & - & 7328 & 7300 & - & 6964 \\
\hline & Exons & $14^{\mathrm{a}}$ & $14^{\mathrm{a}}$ & - & - & 14 & 14 & 14 & - & - & - & 14 & 14 & - & 14 \\
\hline & CDNA & $2706^{a}$ & $2671^{a}$ & - & - & 2667 & 2676 & 2676 & - & - & - & 2586 & 2670 & - & 2529 \\
\hline & Amino acids & $-^{a}$ & $-^{a}$ & - & - & 888 & 891 & 891 & - & - & - & 861 & 889 & - & 842 \\
\hline \multirow[t]{4}{*}{ Gene6 } & DNA & 3986 & 4542 & 3996 & 3987 & 3999 & 4007 & 3958 & - & - & - & 3990 & 3988 & 4294 & 4144 \\
\hline & Exons & 8 & 8 & 8 & 8 & 8 & 8 & 8 & - & - & - & 8 & 8 & 8 & 7 \\
\hline & CDNA & 876 & 873 & 873 & 873 & 873 & 873 & 882 & - & - & - & 882 & 882 & 882 & 891 \\
\hline & Amino acids & 291 & 290 & 290 & 290 & 290 & 290 & 293 & - & - & - & 293 & 293 & 293 & 296 \\
\hline \multirow[t]{4}{*}{ Gene7 } & DNA & 3829 & 3779 & 3782 & 3805 & 3781 & 3775 & 3977 & $3298 \#$ & $1876 \#$ & - & 3814 & 3812 & 3962 & 3717 \\
\hline & Exons & 8 & 8 & 8 & 8 & 8 & 8 & 8 & $7 \#$ & $6 \#$ & - & 8 & 8 & 8 & 8 \\
\hline & CDNA & 1092 & 1092 & 1092 & 1092 & 1092 & 1092 & 1092 & 1023\# & 966\# & - & 1092 & 1092 & 1092 & 1086 \\
\hline & Amino acids & 363 & 363 & 363 & 363 & 363 & 363 & 363 & $340 \#$ & 321\# & - & 363 & 363 & 363 & 361 \\
\hline \multirow[t]{2}{*}{ Gene8 } & DNA & 2988 & 2982 & 2974 & 2991 & $2993^{a}$ & 2991 & 2976 & 2982 & 2993 & - & 2991 & 2991 & 3003 & 3003 \\
\hline & Exons & 5 & 5 & 5 & 5 & $5^{a}$ & 5 & 5 & 5 & 6 & - & 5 & 5 & 5 & 5 \\
\hline
\end{tabular}


Table 5 The feature of syntenic genes on Saccharum and sorghum bacterial artificial chromosome (BAC) clones (Continued)

\begin{tabular}{|c|c|c|c|c|c|c|c|c|c|c|c|c|c|c|c|}
\hline & CDNA & 2412 & 2421 & 2376 & 2415 & $2417^{a}$ & 2415 & 2406 & 2421 & 2367 & - & 2415 & 2415 & 2427 & 2421 \\
\hline & Amino acids & 803 & 806 & 791 & 804 & $-^{a}$ & 804 & 801 & 806 & 788 & - & 804 & 804 & 808 & 806 \\
\hline \multirow[t]{4}{*}{ Gene9 } & DNA & 1632 & 1638 & 1632 & 1626 & - & - & 1632 & 1413 & 1632 & - & 1629 & - & 1575 & $x$ \\
\hline & Exons & 1 & 1 & 1 & 1 & - & - & 1 & 1 & 1 & - & 1 & - & 1 & $x$ \\
\hline & CDNA & 1632 & 1638 & 1632 & 1626 & - & - & 1632 & 1413 & 1632 & - & 1629 & - & 1575 & $x$ \\
\hline & Amino acids & 543 & 545 & 543 & 541 & - & - & 543 & 470 & 543 & - & 542 & - & 524 & $x$ \\
\hline \multirow[t]{4}{*}{ Gene10 } & DNA & $x$ & $x$ & $17423^{a}$ & $22357^{a}$ & - & - & $x$ & $x$ & $620^{a}$ & $744^{\mathrm{a}}$ & $1057^{a}$ & - & 1066 & $x$ \\
\hline & Exons & $x$ & $x$ & $6^{a}$ & $6^{\mathrm{a}}$ & - & - & $x$ & $x$ & $3^{a}$ & $5^{a}$ & $4^{a}$ & - & 6 & $x$ \\
\hline & CDNA & $x$ & $x$ & $490^{\mathrm{a}}$ & $436^{\mathrm{a}}$ & - & - & $x$ & $x$ & $427^{a}$ & $393^{a}$ & $501^{\mathrm{a}}$ & - & 534 & $x$ \\
\hline & Amino acids & $x$ & $x$ & $\mathrm{~N} / \mathrm{A}^{\mathrm{a}}$ & $N / A^{a}$ & - & - & $x$ & $x$ & $N / A^{a}$ & $N / A^{a}$ & $166^{a}$ & - & 177 & $x$ \\
\hline \multirow[t]{4}{*}{ Gene11a } & DNA & $x$ & $x$ & 4273 & 4059 & - & - & $x$ & $x$ & 4043 & 4060 & 4062 & - & $1566 \# / 33480$ & $x$ \\
\hline & Exons & $x$ & $x$ & 7 & 6 & - & - & $x$ & $x$ & 6 & 6 & 6 & - & $4 \# / 6$ & $x$ \\
\hline & cDNA & $x$ & $x$ & 1029 & 1029 & - & - & $x$ & $x$ & 1029 & 1029 & 1029 & - & $645 \# / 993$ & $x$ \\
\hline & Amino acids & $x$ & $x$ & 342 & 342 & - & - & $x$ & $x$ & 342 & 342 & 342 & - & $214 \# / 330$ & $x$ \\
\hline \multirow[t]{4}{*}{ Gene11b } & DNA & 3293 & 3832 & 3671 & 3678 & - & - & 9988 & 3271 & 5684 & 9146 & 3674 & - & 6477 & 4616 \\
\hline & Exons & 6 & 6 & 6 & 6 & - & - & 6 & 6 & 7 & 6 & 6 & - & 7 & 6 \\
\hline & CDNA & 948 & 948 & 948 & 948 & - & - & 948 & 948 & 927 & 948 & 948 & - & 954 & 957 \\
\hline & Amino acids & 315 & 315 & 315 & 315 & - & - & 315 & 315 & 308 & 315 & 315 & - & 317 & 318 \\
\hline \multirow[t]{4}{*}{ Gene12 } & DNA & - & 1836 & - & 1841 & - & - & 2053 & 1840 & 1877 & $1877 / 1881 / 1881^{1}$ & 1841 & - & - & 1846 \\
\hline & Exons & - & 3 & - & 3 & - & - & 3 & 3 & 3 & $3 / 3 / 3$ & 3 & - & - & 3 \\
\hline & cDNA & - & 1536 & - & 1539 & - & - & 1146 & 1536 & 1539 & $1671 / 1611 / 1611$ & 1539 & - & - & 1545 \\
\hline & Amino acids & - & 511 & - & 512 & - & - & 381 & 511 & 512 & $556 / 536 / 536$ & 512 & - & - & 514 \\
\hline
\end{tabular}

Notes: ${ }^{\text {a }}$ pseudo gene \# BAC border $\mathrm{X}$ deletion - out of BACs

1. Three genes of 12 were annotated in the BAC 135 P 16 of R570 
than in all the gene pairs in both S. officinarum haplotypes in LA Purple and S. officinarum-origin haplotypes in the hybrid, which might reflect the reduced functional selective constraint in S. officinarum caused by the duplication of the gene 11a (Fig. 3 and Additional file 7). However, the $\mathrm{Ka} / \mathrm{Ks}$ ratios of gene 11a's pairs were very low in S. officinarum haplotypes, indicating that the gene 11a contributes more critical function to $S$. officinarum than gene 11b. The gene pairs of gene 2, 6 and 12 in haplotypes of S. officinarum and S. officinarum-origin in hybrid had a $\mathrm{Ka} / \mathrm{Ks}$ less than 0.5 , indicating strong selective constraint and their critical function for S. officinarum. (Fig. 3 and Additional file 7).

\section{Neutrality test}

To investigate whether the homologous haplotypes fit the neutral equilibrium model, we performed Tajima's D test for $4 S$. spontaenum (origin) haplotype sequences and $8 S$. officinarum (origin) haplotype sequences, respectively. A significant negative Tajima's D-test statistic indicates an excess of the low frequency of polymorphism, which is consistent with directional selection or population expansion [56]. We observed significant negative Tajima's D value $(-1.00205 ; P<0.001)$ in the homologous regions from $S$. spontaenum, suggesting these regions were under directional selection. While, no significant negative Tajima's $\mathrm{D}$ value (average $=-0.67355, \mathrm{P}>0.1$ ) was observed in the haplotypes from $S$. officinarum (Fig. 5). In addition to Tajima's D, nucleotide diversity (Pi value) was estimated using DnaSP 5.0. We observed significant nucleotide diversity between $S$. spontaneum and $S$. officinarum $($ mean $=$ 0.38 versus mean $=0.54$, Mann-Whitney-Wilcoxon test, $P$ value $<2.2 \times 10-16$ ). More than $60 \%$ of nucleotide diversity for Bru1 lost in the genomic regions of S. spontaneum, whereas Bru1 in S. officinarum kept relatively higher DNA diversity. Fu and Li's D test analysis showed similar results. A negative D value (-0.54) was observed in S. spontaneum. Meanwhile, sliding window (window size $1 \mathrm{kbp}$ and step length $100 \mathrm{bp}$ ) showed that $\mathrm{D}$ values of a number of windows ranging from 11800 to 20300 were significant in S. spontaneum ( $\mathrm{P}$ value $<0.01$ ). However, a positive $\mathrm{D}$ value (0.12269) of $\mathrm{F}$ and $\mathrm{Li}$ was found at Bru1 region in S. officinarum. These results were consistent with directional selection of Bru1 in S. spontaneum.

\section{Comparison of large TEs between the homologous regions of hybrid R570 and its progenitor genome} The sugarcane hybrid R570 BAC sequences have approximately 10-15 \% higher interspersed repeat content $(70.0 \%)$ than in the S. officinarum (59.0\%) and S. spontaneum (55.7\%) BAC sequences (Table 2), which is mainly due to higher Type I transposable element content in R570 than that in S. officinarum and AP85-441 BAC sequences. The AP85-441 BAC sequences have an exceptionally low amount of gypsy type LTR retrotransposons (4.4\%) (Table 2). The Saccharum hybrid R570 BAC sequences have higher content of gypsy type elements $(21.12 \%)$ than copia (11.61 \%), unlike S. spontaneum and S. officinarum BAC sequences that have higher content of copia-type elements (16.24\% and $18.92 \%$ respectively) than gypsy (4.37 \% and $12.06 \%$ ) (Table 2). Additionally, hybrid R570 BAC sequences have more LINE elements (7.32 \%) than S. spontaneum (4.78 \%) and S. officinarum BAC sequences (1.79\%) (Table 2).

The type II TE content in the Bru1 genomic region of the sugarcane hybrid R570 is comparable to its progenitor genomes, though the content of individual families differs. For example, the hybrid R570 BAC sequences have 2.4-2.5 fold lower PIF-Harbinger transposons and 3 to 4 fold higher CACTA/CMC-EnSpm transposons than the sequences of two progenitors. The AP85-441 BAC sequences have about 2.2-2.6 fold higher Tc1-Mariner transposons than in S. officinarum and hybrid R570 BAC sequences, reflecting the differential accumulation of particular $\mathrm{TE}$ subfamilies in S. officinarum, S. spontaneum, and the hybrid.

To study the evolution of the Bru1 locus, the large TEs in the $S$. officinarum and S. spontaneum BAC sequences at the Bru1 genomic region were assessed in comparison with those in the corresponding hybrid R570 BAC sequences (Additional file 6). Four full-length Ty1/copia elements, 2 full-length Ty3/gypsy elements, one full-length $\mathrm{Mu}$-like element, and 10 partial elements (9 retrotransposons and 1 transposon) in the S. officinarum and S. spontaneum BAC sequences were identified (Fig. 4)

The most conserved TE in the Bru1 region is a partial Zn-finger domain ( 38 aa homology to pfam13966: zfRVT) located approximately 102 nucleotides downstream of the coding region of gene 8 (a conserved hypothetical protein). The universal presence of this domain is not only

Table 6 The average sequence identities between the homologous haplotypes from two progenitors and Saccharum hybrids R570

\begin{tabular}{llllllll}
\hline BAC ID & 15 N23 & 197G04 & 142 J21 & 135 P16 & $253 G 12$ & 53A11 & CIR9020/12E03 \\
Haplotype ID & I & III & V & VI & II & IV & VII \\
Genbank ID & FN431663.1 & FN431667.1 & FN431666.1 & FN431665.1 & FN431668.1 & FN431664.1 & FN431669.1/FN431661 \\
Length (bp) & 137851 & 141630 & 126547 & 142236 & 158483 & 81164 & $87631+84926$ \\
S. spontaneum haplotypes & 96.07 & 97.24 & 95.88 & 94.56 & 95.28 & 95.87 & 95.67 \\
S. officinarum haplotypes & 96.61 & 93.82 & 98.38 & 97.48 & 96.65 & 96.62 & 96.41 \\
\hline
\end{tabular}




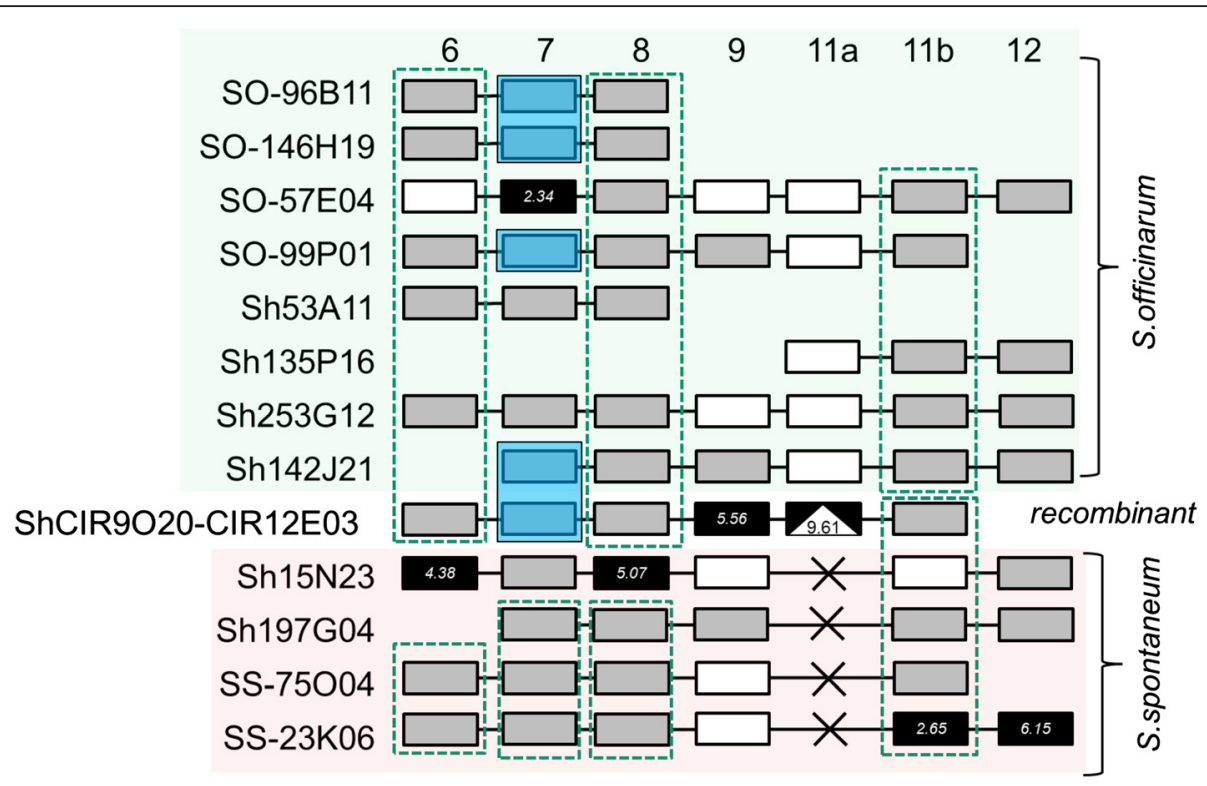

Fig. 2 Schematic representation of verifying the homologous haplotype origin in Saccharum hybrid R570 based on homo(oe)ologous gene allele sequence comparison with two progenitor Saccharum species as references. Notes: Similar analyses as Garsmeur et al. (2011) [18] were performed for the schematic. Each gene allele is represented by a square. For each locus, the most divergent allele is marked in black and its theoretical divergence time (highest estimate observed in Myr) is indicated in italics. All alleles that fall into groups (of at least three) with all values lower than one-third of this maximum divergence time are marked by white squares. When the phylogenetic trees were not degenerate, the alleles (loci $6,7,8$, and 11b) of the same branch (relating to the same internal node) were placed in vertical dotted boxes. But for five alleles of loci 7 , the alleles from the same phylogenetic tree branch were placed in the transparent blue boxes due to they distribute separately in the figure. The white triangle in the black square for locus 11a indicates an insertion. The ' $x$ ' mark indicates absence of the gene

reminiscent of the shared ancestry of this region but is also suggestive of co-option of this derived segment in gene 8. This is supported by the fact that gene 8 mRNA from sorghum (GenBank accession number XM_002453182.1) includes part of this $\mathrm{Zn}$-finger domain.
S. spontaneum clone Ss-23 k06 and hybrid R570 clone Sh197G04 share a retroelement (full-length in both BACs) located between genes 7 and 8. S. officinarum clone So-96B11 and R570 hybrid clone Sh142J21 share 3 retroelements (2 partial TEs and one TE full-length in $S$.

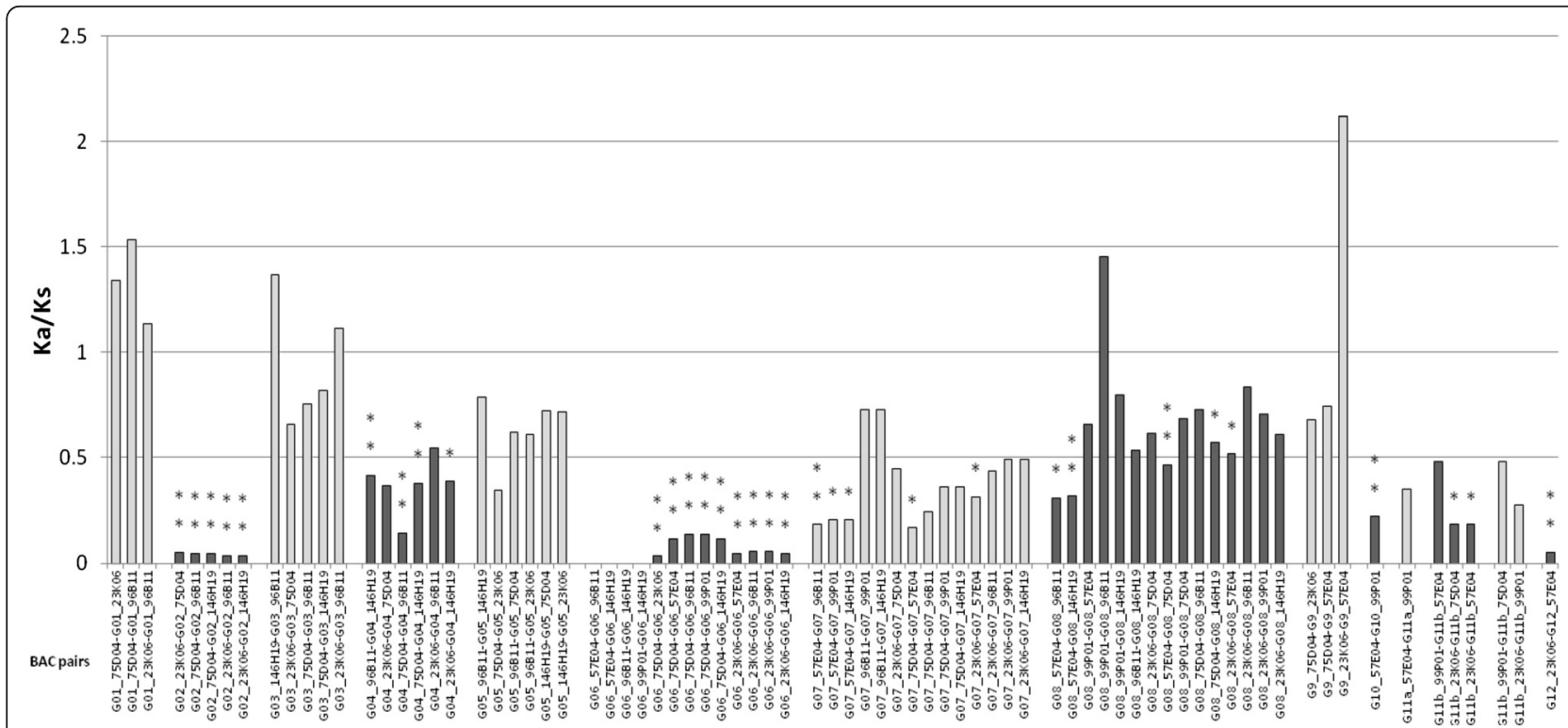

Fig. $3 \mathrm{Ka} / \mathrm{Ks}$ for each gene from homolougs haplotype from S.officinarum (LA Purple) and S.spotaneum(AP85-441). Pairwise comparisons with Duncan's test gave $P$ values of ${ }^{*}, P \leq 0.05 ;{ }^{* *}, P \leq 0.01 ;{ }^{* * *}, P \leq 0.001$ 
officinarum but truncated in hybrid R570 due to its location at the end of BAC) located between genes 8 and gene 9. S. officinarum clones So-57E04 and So-99P01 and the R570 hybrid clone Sh135P16 share two nested TEs (full-length in both of the S. officinarum clones but truncated in the clone from hybrid R570 due to its location at the end of BAC) within the gene 10. R570 clone from Bru1 genomic region was classified into 7 haplotypes [18]. Our results suggest that R570 BACs, Sh142J21 (haplotype V), Sh135P16 (haplotype VI), Sh197G04 clone (haplotype III) were evolved from S. officinarum haplotype, So-96B11, S. officinarum haplotype So-57E04 (and/or possibly So99P01), and S. spontaneum haplotype Ss-23 K06, respectively. In addition to the shared TEs, we identified three full-length TEs and seven partial TEs in S. officinarum or $S$. spontaneum that were not detected or lost at the corresponding location in the hybrid R570 BACs.
Of the nine full-length retrotransposon insertions in the Bru1 locus, six are estimated to be inserted at approximately 1 MYA (ranging from 0.88 to $1.28 \mathrm{MYA}$ ), and two were relatively young (inserted $0.00 \mathrm{Ma}$ and $0.02 \mathrm{Ma}$ ) (Additional file 9). Surprisingly, the insertion time of Ty3/gypsy element $(0.44 \mathrm{Ma})$ within gene 10 of S. officinarum So-99P01 sequence is much shorter than the insertion time of a Ty1/copia (0.99 MYA) nested within this one and also much lower than its counterpart (1.00 MYA) in the other S. officinarum BAC, So-57E04 (Fig. 4). Both TEs in gene 10 of So-99P01 are flanked by intact target site duplications (TSD) and have dispersed mismatches in their respective pairs of LTRs, precluding mis-assembly or localized sequencing errors. Thus, either chance or other factors such as gene conversion may have played a role in the sequence preservation of LTR of this Ty3/gypsy element.

\section{S. officinarum_57E04 \\ S. officinarum_96B11 \\ S. officinarum_99P01 \\ S. officinarum_146h19 \\ R570_Sh 53A11_FN431664.1 \\ R570_Sh 135P16_FN431665.1 \\ R570_Sh 253G12_FN 431668.1 \\ R570_Sh 142J21_FN431666.1 \\ R570_ShCIR9O20_FN431669.1 \\ R570_ShCIR12E03_FN431661.1 \\ R570_Sh 15N23_FN431663.1 \\ R570_Sh 197G04_FN 431667.1 \\ S. spontaneum_208_23k06 \\ S. spontaneum_208_75D04 \\ S. bicolor_24P17c_FN431662.1

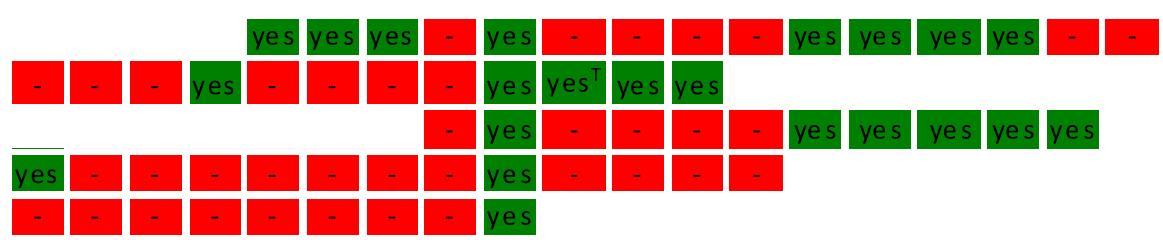 \\ \begin{tabular}{llll|llll}
\hline & - & - & - & - & - & - & - \\
\hline
\end{tabular}
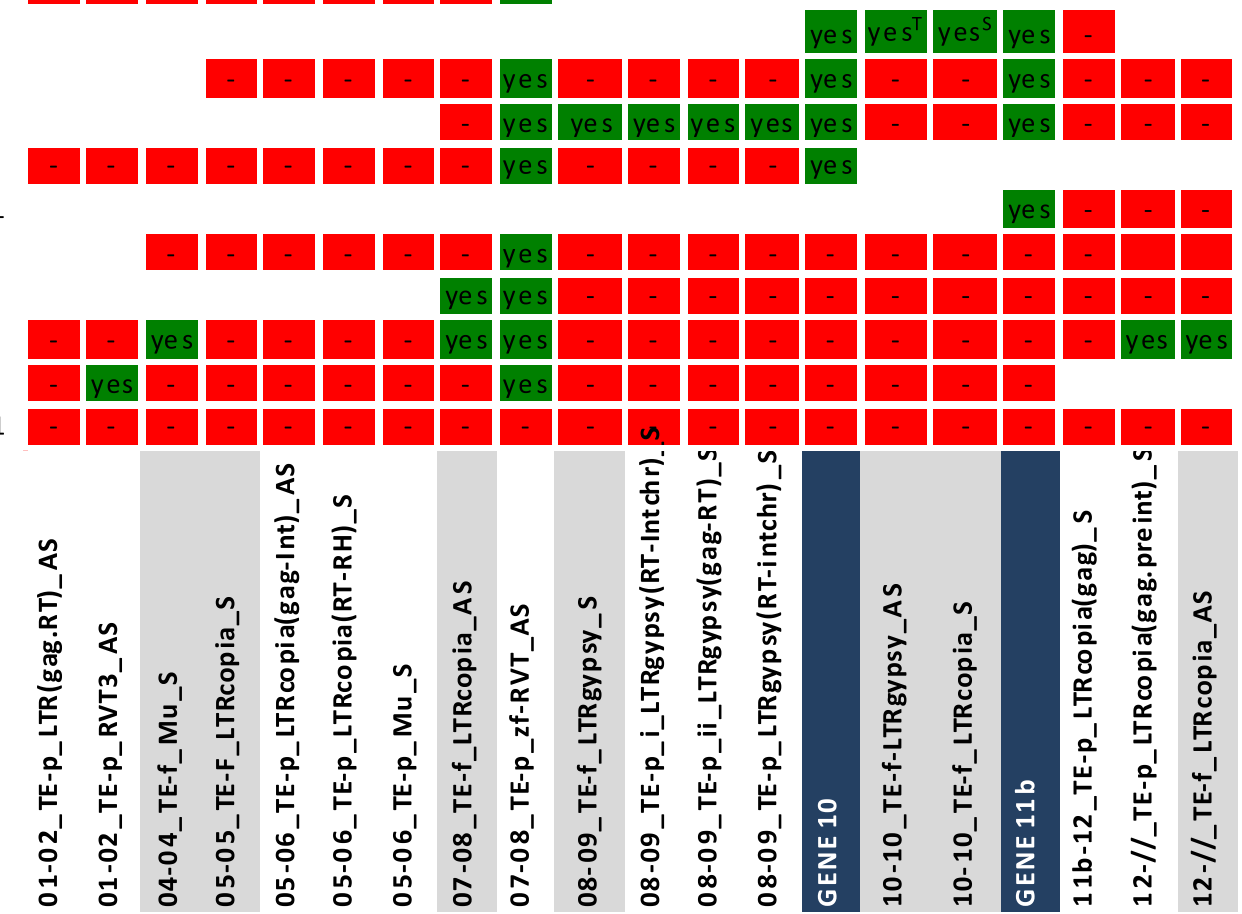

Fig. 4 Distribution of transposable elements (TE) in the Bru1 surrounding regions of LA Purple (S.officinarum), AP85-441 (S. spontaneum), and the hybrid cultivar, R570. Notes: The TE names listed in the bottom row consist of four parts separated by an underscore" -". The first part indicates the location, e.g., 01-02 indicates between gene 1 and gene 2 and 05-05 indicates within gene 5. The second part indicates full length (TE-f) or partial (TE-p). The third part indicates orientation (AS = Antisense, $S=$ Sense). Colum 1 contains the clone identifiers: the species name, BAC name, and accession (if any) separated by underscore and the remaining columns score the presence (green) or absence (red) of each TEs listed at the bottom. A superscript "T" indicates a likely full length TE that was truncated due to its presence at the end of BAC and a superscript "S" indicates a solo LTR. The presence and absence of gene 10 (dark blue highlight in bottom row) and gene $11 \mathrm{~b}$ (dark blue highlight) is also given for reference because these two genes are present in S. officinarum BAC sequences but absent in S. spontaneum BAC sequences. The blank cells in white indicate no data available 


\section{Discussions}

Modern sugarcane cultivars are developed from hybridization between $S$. officinarum with high sugar content and S. spontaneum with stress tolerance. Limited genetic diversity of parental clones became the bottleneck for modern sugarcane breeding. Identification of haplotypes of the main Saccharum species and tracing their evolutionary history after hybridization will provide essential information for sugarcane improvement. The isolation and sequencing of BACs in the genomic regions of the rust resistance gene in S. officinarum and $S$. spotaneum offered an opportunity to study the genomic features of the progenitor species in these fast-evolving and agronomically important sequences, and to validate the prediction of haplotype origins in hybrid R570.

Although haplotypes were highly conserved within and between Saccharum species, our study identified species-specific insertions and deletions, which likely occurred after the speciation event, and can be used to identify origins of haplotypes in modern sugarcane hybrids. Interestingly, genes 10 and 11a were completely missing in S. spontaneum, which might attribute to the consequence of the speciation event. Multiple alleles in autopolyploids reduced selective constraint for those genes with no advantage in higher dose, and some alleles could have undergone pseudogenization. In the genomic region of rust resistant genes, 6 out of 52 gene alleles became pseudogenes. Out of the 15 paired alignments, InDels broke down alignments in $32.18 \%$ sequences, which caused a frame shift and introduced premature stop codons in some alleles and made them pseudogenes.
Comparison of haplotype sequences within species showed that S. spontaneum had larger haplotype variations than that of $S$. officinarum (Additional file 10), suggesting earlier polyploidization in S. spontaneum than in S. officinarum, which could have contributed to or even caused the speciation event leading to the divergence of $S$. spontaneum from the rest of Saccharum species. In general, the sequence divergence is the highest between $S$. spontaneum and $S$. officinarnum, medium among S. spontaneum haplotypes, and the lowest among the $S$. officinarnum haplotypes.

Saccharum species had undergone extensive genome rearrangements following polyploidization in the Bru1 region, which is similar to the instability of maize genome after polyploidization [57]. Gene $11 \mathrm{a}$ and gene $11 \mathrm{~b}$ are duplicated genes in S. officinarum. Gene11b existed in all of the $S$. officinarum haplotypes but was missing in S. spontaneum and sorghum (Fig. 1 and Table 5). Phylogenetic analyses showed that gene $11 \mathrm{~b}$ was closer to its homologs in sorghum and rice than the 11a observed in S.officinarum (Additional file 11), which indicated a duplication event of gene 11 occurred after the speciation event separating Saccharum and Sorghum. Due to the absence of gene $11 \mathrm{~b}$ in $S$. spontaneum genome, the duplication event of gene 11 likely occurred after the speciation event of S. officinarum and S. spontaneum and could be lineage specific in S. officinarum.

Comparing to Bru1 region in Saccharum species, no large TEs were observed between the genes of the corresponding region in sorghum (Fig. 4). The large TEs between genes in Adh1 region of Saccharum hybrids were also absent in the corresponding region of sorghum
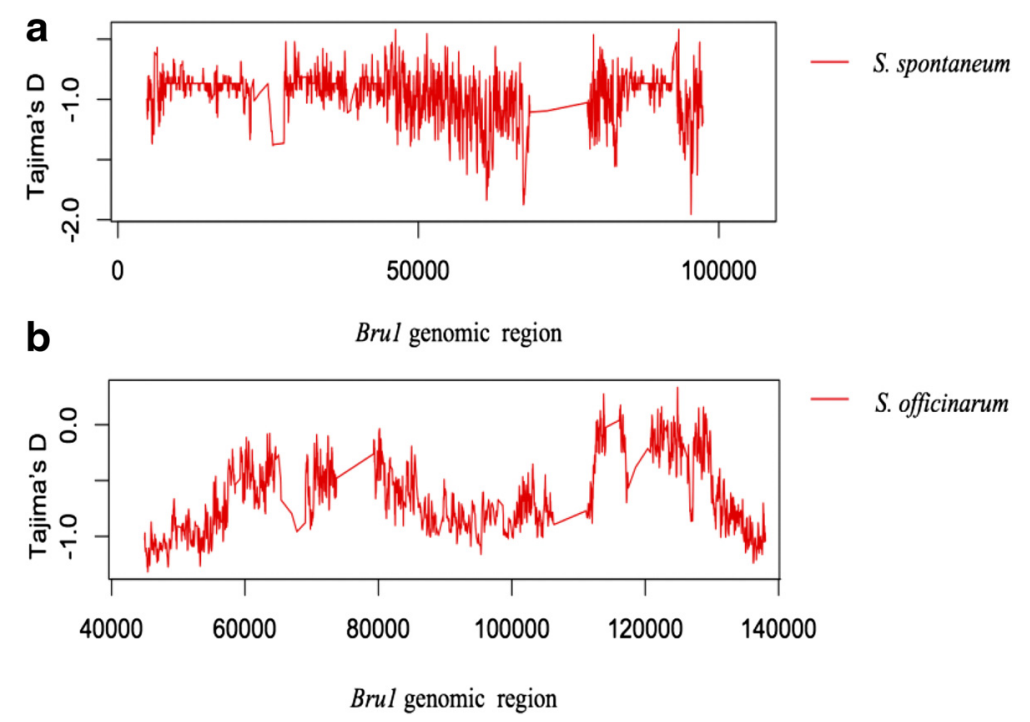

Fig. 5 Tajima'D test for brul genomic regions from S. spontaneum (a) and S. officinarum (b). Notes: Homologous haplotype sequences in S. spontaneum include Sh15N23, Sh197G04, Ss-75004 and Ss-23 K06. Homologous haplotype sequences in S. officinarum contain So-96B11, So-146H19, So-57E04, So-99P01, Sh53A11, Sh135P16, Sh253G12 and Sh142J21 
genome [58]. A TE zf-RVT in the Brul regions of Saccharum was speculated to derived from gene 8 (Fig. 4), indicating small fragment duplication occurred before the polyploidization of Saccharum and after the separation between Saccharum and Sorghum. Moreover, TEs are more abundant in S. officinarum (59.04 \%) than in S. spontaneum (55.68 \%) (Table 2). These results suggested that genome expansion in this region of S. officinarum, compared to S. spontaneum, was caused by TE accumulation.

Modern sugarcane hybrids contain estimated 8-14 copies of homologous chromosomes, and can have up to 14 different alleles [59]. Although multiple alleles are considered to be functionally redundant at the time of origin, they provide raw materials for the evolution of novelty by relaxing purifying selection on the duplicated genes [60-63]. Six out of 51 allelic genes became pseudogenes in Saccharum, likely due to functional redundancy. S. spontaneum genome had undergone more dynamic genome rearrangement than $S$. officinarum genome. In paleopolyploids [64-67], and recent allopolyploid species, such as wheat $[68,69]$ and Tragopogon $[70,71]$, eliminations and pseudogenizations of essential functional genes have been well documented.

Among the 13 unique genes, 451 allele pairs from Saccharum species, Saccharum hybrids and sorghum were used for $\mathrm{Ka} / \mathrm{Ks}$ analysis, excluding genes that were missing in the $S$. spontaneum haplotypes. The $\mathrm{Ka} / \mathrm{Ks}$ ratio of $94.5 \%$ allele pairs (428 pairs) was less than 1, suggesting that the majority of homologous alleles were under purifying selection, which is consistent with the $\mathrm{Ka} / \mathrm{Ks}$ ratios of genes in haplotypes of hybrid R570 [18]. Selection resulted in nonrandom radical amino acid substitutions for many genes [72]. Our results verified that gene 11a was a $S$. officinarum specific gene and likely a recent duplication from gene $11 \mathrm{~b}$, resulting a lower $\mathrm{Ka} / \mathrm{Ks}$ ratio.

Gene structures are highly conserved in Bru1 and Adh1 regions among haplotypes within the R570 genome $[18,58]$. InDels were found in introns of genes when compared to sorghum and introns exhibited more variations than exons as expected [58]. In our study, except for genes 1,2 , and 9 , the other 10 unique genes exhibited variable sizes of introns among the homologous alleles (Additional file 10), which are resources for developing intron length polymorphism markers in sugarcane. Variations in introns have been associated with biological function both in animal and plant $[73,74]$. Insertions of LTRs were found in introns of genes 4, 10,11a and 11b, some of which could have evolved new functions via neofunctionalization, or partition their ancestral roles via subfunctionalization. Intron gain/ loss events were found in hybrid R570, such as gene 1 in ShIV (Sh53A11), gene 8 in ShV (Sh15N23) (Fig. 4). Intron gain/loss is not a commonly ongoing process, but rather triggered by certain dramatic evolutionary events that lead to long-term bottlenecks [75]. Since intron gain/loss events were only observed in the hybrid genome, they might have been triggered by hybridization event.

To discriminate the origin of the genome in the hybrids, we performed three comparative analyses: sequence similarity, species-specific InDels, and gene phylogenetic combined with haplotype networks. Sequence comparison among the homologous haplotype could be used to identify the origin of most regions in Saccharum hybrids genome, but may not be sufficient to discriminate the recombinant haplotype due to the little divergence between S. officinarum and S. spontaneum. Species-specific InDels could be reliable markers for identifying the origin of Saccharum hybrid genome. Further comparison of genome between $S$. officinarum and S. spontaneum may identify the InDels for discriminating the recombinant genome in Saccharum hybrids. Gene phylogenetic and haplotype network analysis could be used for identifying recombinant genome in Saccharum hybrids(Fig. 2).

One of the major challenges for sequencing sugarcane hybrid cultivar genomes is to distinguish the fractions from S. spontaneum, S. officinarum and the recombinant genome. We performed the sequence analysis of homologous haplotypes from the progenitor species of Saccharum hybrids surrounding the Brul region. Retrotransposon insertions and sequences variations among the homologous haplotypes sequence divergence ranged from $18.2 \%$ to $60.5 \%$ with an average of $33.7 \%$, comparable to the 12.8 $23.3 \%$ InDels divergence among homologous chromosomes in hexaploid wheat, which allows sequencing and assembling the autopolyploid Saccharum genomes and the auto-allopolyploid hybrid genomes using whole genome shotgun sequencing approach as demonstrated in wheat [76, 77]. However, long read sequencing would be necessary for discriminate the homologous haplotypes in the progenitor species because identical fragments of short reads exist in Saccharum genomes.

The genome sequence diversity in wild species $S$. spontaneum have been demonstrated to be greater than that of the domesticated species S. officinarum [78-81]. However, the two S. spontaneum homologous sequences (Ss-75D04 and Ss-23 K06) shared higher similarity(98 \%) than the sequence similarities among the homologous haplotype sequences from S. officinarum.Moreover, $S$. spontaneum-originated hapotypes were under directional selection with an average windowed Tajima's D value of $-1.00205(p<0.001)$, while, the $S$. officinarum haplotypes showed no significant negative Tajima's D value. S. spontaneum contributed stress tolerance for Saccharum cultivar hybrid (Fig. 5). These results suggested that the Brul genomic region in Saccharum hybrid originated from S. spontaneum and is under strong directional selection. The candidate bru1 gene is supposed to be under strong functional constraint and has a more substantial selection in $S$. spontaneum than that in $S$. 
officinarum. Of 10 homologus genes in the Bru1 genomic haplotype regions, 5 (gene 2, 4, 6, 7 and 8) were revealed to under strong functional constraint based on the $\mathrm{ka} / \mathrm{ks}$ analysis, but none of them were found to have a higher selection in S. spontaneum than in S. officinarum. The bru1 gene in $\mathrm{R} 570$ was revealed to be single-dose [13], it is challenge to predicted dosage of bru1 gene in S.spontaneum, but we can conclude that bru1 is not octopi-dosage as the bru1 in R570 was single-dose. Therefore, the bru1 gene may not existed in the two haplotype sequences from $S$. spontaneum as only quarter of alleles were sequenced, but it could be presented in the S.spontaneum originated haplotype within R570 because 2 alleles from S.spontaneum may cover all the $S$. spontaneum originated alleles in the Saccharum hybrid. Map-based cloning is hard to refine the gene in such limited genomic region. To further identify the bru1 gene, gene expression experiment based on RNA-seq could be used to test the expressional level of the functional constraint genes. Candidate gene transformation would be necessary to final confirm the bru1 genes.

\section{Conclusions}

The commercial sugarcane cultivars are complicated allo-autopolyploid and aneuploid, mostly derived from interspecific hybridization between S. spontaneum and S.officinarum. This study reports the first comparison among haplotypes of a modern sugarcane hybrid R570 and its progenitor species, and provides new insights into the genome evolution of modern sugarcane cultivars. With the homologous sequences from the two progenitor species as references, species-specific InDels were identified, which were used to validate the species origins of the 7 haplotypes from the hybrid genome of R570. Our results confirmed that 4 haplotypes of R570 were originated from $S$. officinarum, 2 from $S$. spontaneum. ShCIR9020-CIR12E03 was originated from recombination. Tajima's $\mathrm{D}$ analysis showed that $S$. spontaneum haplotypes in Brul genomic region were under directional selection, contributing to brown rust resistance in Saccharum hybrid. The duplication event of gene 11 and InDels among the homologous haplotypes suggested that Saccharum species had undergone genome rearrangements after speciation. Gene content and gene structure were relatively well conserved among the homologous haplotypes. Exon splitting occurred in haplotypes of the hybrid genome but not in its progenitor genomes, signalling genome rearrangements after hybridization. Among all the homologous alleles, introns vary in size while the exons are conserved. Pseudogenes (alleles) caused by InDels were observed for all annotated genes except gene 10 in the two Saccharum species.

\section{Additional files}

Additional file 1: Table S1. The sequences of primers used for probe preparation for the BAC library screening. (DOCX 15 kb)

Additional file 2: Table S2. List of syntenic genes of the BAC clone sequences (DOCX $19 \mathrm{~kb}$ )

Additional file 3: Figure S1. Pairwise comparision of BAC sequences from LA Purple (S.officinarum), AP85-441 (S. spontaneum), Saccarhum Hybrids(R570) (DOCX 497 kb)

Additional file 4: Figure S2. Mauve visualization of local collinear blocks identified among 14 haplotypes (15BACs) from Saccharum species and sorghum. (DOCX $1909 \mathrm{~kb}$ )

Additional file 5: Figure S3. Gene structure comparison of different haplotype sequences from LA Purple (S. officinarum), AP85-441 (S. spontaneum), and the hybrid cultivar, R570. (DOCX $700 \mathrm{~kb}$ )

Additional file 6: Figure S4. The genome structure of haplotypes surrounding the Bru1 locus from LA Purple (S. officinarum), AP85-441 (S. spontaneum), and the hybrid cultivar, R570. (DOCX 95 kb)

Additional file 7: Table S3. Estimation of synonymous and nonsynonymous nucleotide divergence among S. officinarum (LA Purple), S. spontaneum(AP85-441) and hybrid cultivar R570. (DOCX 24 kb)

Additional file 8: Table S4. The average $\mathrm{Ka} / \mathrm{Ks}$ ratio of the gene pairs within and between LA Purple (S. officinarum), AP85-441 (S. spontaneum), and the hybrid cultivar, R570. (DOCX 16 kb)

Additional file 9: Table S5. Estimated insertion time of full length retrotransposons in Bru1 locus of LA Purple (S. officinarum), AP85-441 (S. spontaneum), and the hybrid cultivar, R570. (DOCX 18 kb)

Additional file 10: Table S6. Summary of the gapless comparisons of pairs of BAC clone sequences from LA Purple (S. officinarum), AP85-441 (S. spontaneum), and the hybrid cultivar, R570. (DOCX 39 kb)

Additional file 11: Figure S5. Phylogenetic analysis of 14 haplotypes of genes 11 from Saccharum species, and its homologs from sorghum, Zea mays, and rice. The tree was constructed by the neighbor-joining method implemented in MEGA4 software. The robustness of the tree topology was assessed with 1000 bootstrap replicates. The coding sequences of S6PDH from Zea mays and Malus domestica were used to root the tree. (DOCX 108 kb)

\section{Abbreviations}

BAC, bacterial artificial chromosome; $\mathrm{CM}$, centimorgan; TE, transposable element; SNP, single nucleotide polymorphism; InDels, insertion/deletion; MYA, million years ago; LTRs, long terminal repeat; RFLP, restricted fragment length polymorphism; MITE, miniature inverted transposable element; LINE, long interspersed element; CDD, conserved domain database; TSD, target site duplication

\section{Acknowledgements}

We thank Mr. Muhammad Umar for language editing.

Funding

This project was supported by grants from the 863 program

(2013AA100604), the International Consortium for Sugarcane Biotechnology, EBI BP2012002J17, and US DOE DE-SC0010686.

\section{Availability of data and material}

The sequences of the six clones were deposited in Genbank (accession numbers: KP063111- KP063116). Phylogenetic data for Additional file 11 (alignments and phylogenetic trees) have been deposited to TreeBase and are accessible via the URL: http://purl.org/phylo/treebase/phylows/study/ TB2:S19321.

\section{Authors' contributions}

RM and JZ conceived the study and designed the experiments. JZ, QY and $L Z$ carried out the experiments. JZ, AS, QY, JW, LL, XZ, and YC analyzed the data. JZ and RM wrote the manuscript. All authors read and approved the final manuscript. 


\section{Authors' information}

Not applicable.

\section{Competing interests}

The authors declare that they have no competing interests.

\section{Consent for publication}

Not applicable.

\section{Ethics approval and consent to participate}

Not applicable.

\section{Author details}

${ }^{1}$ FAFU and UIUC-SIB Joint Center for Genomics and Biotechnology, Fujian Provincial Key Laboratory of Haixia Applied Plant Systems Biology, Haixia Institute of Science and Technology, Fujian Agriculture and Forestry University, Fuzhou 350002, Fujian Province, China. ${ }^{2}$ College of Life Sciences, Fujian Normal University, Fuzhou 350108, China. ${ }^{3}$ Department of Plant Biology, University of Illinois at Urbana-Champaign, Urbana, IL 61801, USA. ${ }^{4}$ Texas A\&M AgriLife Research, Department of Plant Pathology \& Microbiology, Texas A\&M University System, 17360 Coit Road, Dallas, TX 75252, USA. ${ }^{5}$ Department of Agronomy, University of Florida, 2033 Mowry Road, Gainesville, FL 32610, USA. ${ }^{6}$ College of Horticulture, Nanjing Agricultural University, 1 Weigang Road, Nanjing 210095, China. ${ }^{7}$ College of Plant Science, Jilin University, Changchun, Jilin 130062, China.

Received: 5 December 2015 Accepted: 7 June 2016 Published online: 10 June 2016

\section{References}

1. D'Hont A, Grivet L, Feldmann P, Glaszmann JC, Rao S, Berding N. Characterisation of the double genome structure of modern sugarcane cultivars (Saccharum spp.) by molecular cytogenetics. Molecular and General Genetics MGG. 1996;250(4):405-13. \%@ 0026-8925.

2. Price S. Cytogenetics of modern sugar canes. Economic Botany. 1963;17(2): 97-106.\%@0013-0001.

3. Price S. Interspecific hybridization in sugarcane breeding, vol. 1965. 1965. p. 1021-6.

4. Berding N, Roach BT. Germplasm collection, maintenance, and use, vol. 143 Amsterdam: Sugarcane improvement through breeding Elsevier; 1987. p. 210

5. Grivet L, D'Hont A, Roques D, Feldmann P, Lanaud C, Glaszmann JC. RFLP mapping in cultivated sugarcane. In: Saccharum. 1996.

6. Hoarau JY, Grivet L, Offmann B, Raboin LM, Diorflar JP, Payet J, Hellmann M, D'Hont A, Glaszmann JC. Genetic dissection of a modern sugarcane cultivar (Saccharum spp.). II. Detection of QTLs for yield components. Theor Appl Genet. 2002;105(6-7):1027-37.\%@ 0040-5752.

7. Piperidis G, D'Hont A, Hogarth DM. 2001: Chromosome composition analysis of various Saccharum interspecific hybrids by genomic in situ hybridisation (GISH). Brisbane, Australia: Australian Society of Sugar Cane Technologists; 2001. p. 565-6.\%@0949678104

8. Cuadrado A, Acevedo R, De La Espina SMD, Jouve N, De La Torre C. Genome remodelling in three modern S. officinarum $\times$ S. spontaneum sugarcane cultivars. J Exp Bot. 2004;55(398):847-54. \%@ 0022-0957.

9. Bremer G. Problems in breeding and cytology of sugar cane. Euphytica. 1961:10(1):59-78.\%@0014-2336.

10. Tomkins JP, Yu Y, Miller-Smith H, Frisch DA, Woo SS, Wing RA. A bacterial artificial chromosome library for sugarcane. Theor Appl Genet. 1999;99(3-4): 419-24.\%@0040-5752.

11. D'hont A. Unraveling the genome structure of polyploids using FISH and GISH; examples of sugarcane and banana. Cytogenet Genome Res. 2005; 109(1-3):27-33.\%@ 1424-1859X.

12. Zhang J, Nagai C, Yu Q, Pan Y-B, Ayala-Silva T, Schnell RJ, Comstock JC, Arumuganathan AK, Ming R. Genome size variation in three Saccharum species. Euphytica. 2012;185(3):511-9.\%@ 0014-2336.

13. Daugrois JH, Grivet L, Roques D, Hoarau JY, Lombard H, Glaszmann JC, d'Hont A. A putative major gene for rust resistance linked with a RFLP marker in sugarcane cultivar 'R570'. Theor Appl Genet. 1996;92(8):1059-64. \%@0040-5752.

14. Asnaghi C, D'Hont A, Glaszmann JC, Rott P. Resistance of Sugarcane Cultivar R 570 to Puccinia melanocephala Isolatesfrom Different Geographic Locations. Plant Disease. 2001;85(3):282-6. \%@ 0191-2917.
15. Asnaghi C, Paulet F, Kaye C, Grivet L, Deu M, Glaszmann JC, D'Hont A. Application of synteny across Poaceae to determine the map location of a sugarcane rust resistance gene. Theor Appl Genet. 2000;101(5-6):962-9. $\% @ 0040-5752$.

16. Asnaghi C, Roques D, Ruffel S, Kaye C, Hoarau JY, Telismart H, Girard JC, Raboin LM, Risterucci AM, Grivet L. Targeted mapping of a sugarcane rust resistance gene (Bru1) using bulked segregant analysis and AFLP markers. Theor Appl Genet. 2004;108(4):759-64. \%@ 0040-5752.

17. Le Cunff L, Garsmeur O, Raboin LM, Pauquet J, Telismart H, Selvi A, Grivet L, Philippe R, Begum D, Deu M. Diploid/polyploid syntenic shuttle mapping and haplotype-specific chromosome walking toward a rust resistance gene (Bru1) in highly polyploid sugarcane (2n 12x 115). Genetics. 2008;180(1): 649-60.\%@0016-6731.

18. Garsmeur O, Charron C, Bocs S, Jouffe V, Samain S, Couloux A, Droc G, Zini C, Glaszmann JC, Van Sluys MA. High homologous gene conservation despite extreme autopolyploid redundancy in sugarcane. New Phytologist. 2011:189(2):629-42.\%@1469-8137.

19. Fitch MM, Moore PH. Production of haploid Saccharum spontaneum L.-comparison of media for cold incubation of panicle branches and for float culture of anthers. J Plant Physiol. 1984;117(2):169-78. \%@ 0176-1617.

20. Ming R, Moore PH, Zee F, Abbey CA, Ma H, Paterson AH. Construction and characterization of a papaya BAC library as a foundation for molecular dissection of a tree-fruit genome. Theor Appl Genet. 2001;102(6-7):892-9. $\% @ 0040-5752$

21. Yu Q, Guyot R, de Kochko A, Byers A, Navajas-Pérez R, Langston BJ, Dubreuil-Tranchant C, Paterson AH, Poncet V, Nagai C. Micro-collinearity and genome evolution in the vicinity of an ethylene receptor gene of cultivated diploid and allotetraploid coffee species (Coffea). Plant J. 2011;67(2):305-17.\%@ 1365-1313X

22. Ouyang S, Buell CR. The TIGR Plant Repeat Databases: a collective resource for the identification of repetitive sequences in plants. Nucleic Acids Res. 2004:32 suppl 1:D360-3.\%@ 0305-1048.

23. Nussbaumer T, Martis MM, Roessner SK, Pfeifer M, Bader KC, Sharma S, Gundlach H, Spannagl M. MIPS a database framework for comparative plant genome research. Nucleic Acids Res. 2013;41(D1):D1144-51. \%@ 0305-1048.

24. Jurka J, Kapitonov W, Pavlicek A, Klonowski P, Kohany O, Walichiewicz J. Repbase Update, a database of eukaryotic repetitive elements. Cytogenet Genome Res. 2005;110(1-4):462-7. \%@ 1424-1859X.

25. Chen J, Hu Q, Zhang Y, Lu C, Kuang H: P-MITE: a database for plant miniature inverted-repeat transposable elements. Nucleic acids research 2013

26. Swarbreck D, Wilks C, Lamesch P, Berardini TZ, Garcia-Hernandez M, Foerster H, Li D, Meyer T, Muller R, Ploetz L. The Arabidopsis Information Resource (TAIR): gene structure and function annotation. Nucleic Acids Res. 2008;36 suppl 1:D1009-14.\%@0305-1048.

27. Vogel JP, Garvin DF, Mockler TC, Schmutz J, Rokhsar D, Bevan MW, Barry K, Lucas S, Harmon-Smith M, Lail K. Genome sequencing and analysis of the model grass Brachypodium distachyon. Nature. 2010;463(7282):763-8. \%@ 0028-0836.

28. Ouyang S, Zhu W, Hamilton J, Lin H, Campbell M, Childs K, Malek RL, Lee A, Zheng L, Orvis J. The TIGR Rice Genome Annotation Resource: improvements and new features. Nucleic Acids Res. 2007;35:D883-887.

29. Bennetzen JL, Schmutz J, Wang H, Percifield R, Hawkins J, Pontaroli AC, Estep M, Feng L, Vaughn JN, Grimwood J. Reference genome sequence of the model plant Setaria. Nat Biotechnol. 2012;30(6):555-61.\%@ 1087-0156.

30. Schnable PS, Ware D, Fulton RS, Stein JC, Wei F, Pasternak S, et al. The B73 maize genome: complexity, diversity, and dynamics. Science. 2009; 326(5956):1112-5. \%@ 0036-8075.

31. Paterson AH, Bowers JE, Bruggmann R, Dubchak I, Grimwood J, Gundlach H, Haberer G, Hellsten U, Mitros T, Poliakov A. The Sorghum bicolor genome and the diversification of grasses. Nature. 2009:457(7229):551-6. \%@ 0028-0836.

32. Cantarel BL, Korf I, Robb SMC, Parra G, Ross E, Moore B, Holt C, Alvarado AS, Yandell M. MAKER: an easy-to-use annotation pipeline designed for emerging model organism genomes. Genome Res. 2008;18(1):188-96. \%@ 1088-9051.

33. Llorens C, Futami R, Covelli L, Domínguez-Escribá L, Viu JM, Tamarit D, Aguilar-Rodríguez J, Vicente-Ripolles M, Fuster G, Bernet GP: The Gypsy Database (GyDB) of mobile genetic elements: release 2.0. Nucleic acids research 2010:gkq1061 \%@ 0305-1048

34. Smit AFA, Hubley R, Green P. RepeatMasker Open-3.0. 1996.

35. Flutre $T$, Duprat $E_{1}$ Feuillet $C$, Quesneville $H$. Considering transposable element diversification in de novo annotation approaches. PLoS One. 2011;6(1):e16526.\%@ 11932-16203.

36. Smit AFA, Hubley R. RepeatModeler Open-1.0. 2008. 
37. Li W, Godzik A. Cd-hit: a fast program for clustering and comparing large sets of protein or nucleotide sequences. Bioinformatics. 2006;22(13):1658-9. $\% @ 1367-4803$.

38. Fu L, Niu B, Zhu Z, Wu S, Li W. CD-HIT: accelerated for clustering the nextgeneration sequencing data. Bioinformatics. 2012;28(23):3150-2. \%@ 1367-4803.

39. Han Y, Wessler SR: MITE-Hunter: a program for discovering miniature inverted-repeat transposable elements from genomic sequences. Nucleic acids research 2010:gkq862 \%@ 0305-1048

40. Marchler-Bauer A, Zheng C, Chitsaz F, Derbyshire MK, Geer LY, Geer RC, Gonzales NR, Gwadz M, Hurwitz DI, Lanczycki CJ: CDD: conserved domains and protein three-dimensional structure. Nucleic acids research 2012: gks1243\%@0305-1048

41. SanMiguel P, Gaut BS, Tikhonov A, Nakajima Y, Bennetzen JL. The paleontology of intergene retrotransposons of maize. Nat Genet. 1998;20(1):43-5.

42. Edgar RC. MUSCLE: multiple sequence alignment with high accuracy and high throughput. Nucleic Acids Res. 2004;32(5):1792-7. \%@ 0305-1048.

43. Tamura K, Stecher G, Peterson D, Filipski A, Kumar S: MEGA6: molecular evolutionary genetics analysis version 6.0. Molecular biology and evolution 2013:mst197\%@0737-4038

44. Ma J, Bennetzen JL. Rapid recent growth and divergence of rice nuclear genomes. Proc Natl Acad Sci U S A. 2004;101(34):12404-10. \%@ 10027-18424.

45. Milne I, Bayer M, Cardle L, Shaw P, Stephen G, Wright F, Marshall D. Tablet—next generation sequence assembly visualization. Bioinformatics. 2010:26(3):401-2.\%@1367-4803.

46. Mortazavi A, Williams BA, McCue K, Schaeffer L, Wold B. Mapping and quantifying mammalian transcriptomes by RNA-Seq. Nat Methods. 2008;5(7): 621-8.\%@1548-7091.

47. Larkin MA, Blackshields G, Brown NP, Chenna R, McGettigan PA, McWilliam H, Valentin F, Wallace IM, Wilm A, Lopez R. Clustal W and Clustal X version 2.0. Bioinformatics. 2007;23(21):2947-8.\%@ 1367-4803.

48. Suyama M, Torrents D, Bork P. PAL2NAL: robust conversion of protein sequence alignments into the corresponding codon alignments. Nucleic Acids Res. 2006;34 suppl 2:W609-12.\%@ 0305-1048.

49. Wang D, Zhang Y, Zhang Z, Zhu J, Yu J. KaKs_Calculator 2.0: a toolkit incorporating gamma-series methods and sliding window strategies. Genomics Proteomics Bioinformatics. 2010;8(1):77-80. \%@ 1672-0229.

50. Yang Z, Nielsen R. Estimating synonymous and nonsynonymous substitution rates under realistic evolutionary models. Mol Biol Evol. 2000; 17(1):32--43.\%@0737-4038.

51. F DM. Agricolae: statistical procedures for agricultural research.R package version 1. 2014.

52. Sullivan MJ, Petty NK, Beatson SA. Easyfig: a genome comparison visualizer. Bioinformatics. 2011:27(7):1009-10.\%@ 1367-4803.

53. Schwartz S, Kent WJ, Smit A, Zhang Z, Baertsch R, Hardison RC, Haussler D, Miller W. Human-mouse alignments with BLASTZ. Genome Res. 2003;13(1): 103-7.\%@1088-9051.

54. Carver TJ, Rutherford KM, Berriman M, Rajandream M-A, Barrell BG, Parkhill J. ACT: the Artemis comparison tool. Bioinformatics. 2005;21(16):3422-3. $\% @ 1367-4803$.

55. Darling ACE, Mau B, Blattner FR, Perna NT. Mauve: multiple alignment of conserved genomic sequence with rearrangements. Genome Res. 2004; 14(7):1394-403.\%@ 1088-9051.

56. Tajima F. Statistical method for testing the neutral mutation hypothesis by DNA polymorphism. Genetics. 1989;123(3):585-95. \%@ 0016-6731.

57. Ilic K, SanMiguel PJ, Bennetzen JL. A complex history of rearrangement in an orthologous region of the maize, sorghum, and rice genomes. Proc Natl Acad Sci. 2003;100(21):12265-70. \%@ 10027-18424.

58. Jannoo N, Grivet L, Chantret N, Garsmeur O, Glaszmann JC, Arruda P, D'Hont A. Orthologous comparison in a gene-rich region among grasses reveals stability in the sugarcane polyploid genome. Plant J. 2007:50(4):574-85. \%@ 1365-1313X.

59. Aitken $\mathrm{KS}$, Jackson PA, McIntyre CL. A combination of AFLP and SSR markers provides extensive map coverage and identification of homo (eo) logous linage groups in a sugarcane cultivar. Theor Appl Genet. 2005;110(5):789-801.\%@ 0040-5752.

60. Ohno S. Evolution by gene duplication. Berlin, New York: Springer-Verlag; 1970. p. 160.

61. Prince VE, Pickett FB. Splitting pairs: the diverging fates of duplicated genes. Nat Rev Genet. 2002:3(11):827-37.\%@ 1471-0056.
62. Lynch M, Conery JS. The origins of genome complexity. Science. 2003; 302(5649):1401-4.\%@ 0036-8075.

63. Flagel LE, Wendel JF. Gene duplication and evolutionary novelty in plants. New Phytologist. 2009:183(3):557-64. \%@ 1469-8137.

64. Blanc G, Wolfe KH. Widespread paleopolyploidy in model plant species inferred from age distributions of duplicate genes. Plant Cell. 2004;16(7): 1667-78.\%@1532-1298X.

65. Thomas BC, Pedersen B, Freeling M. Following tetraploidy in an Arabidopsis ancestor, genes were removed preferentially from one homeolog leaving clusters enriched in dose-sensitive genes. Genome Res. 2006;16(7):934-46. \%@1088-9051.

66. Freeling M. Bias in plant gene content following different sorts of duplication: tandem, whole-genome, segmental, or by transposition. Annu Rev Plant Biol. 2009;60:433-53. \%@ 1543-5008.

67. Throude M, Bolot S, Bosio M, Pont C, Sarda X, Quraishi UM, Bourgis F, Lessard P, Rogowsky P, Ghesquiere A. Structure and expression analysis of rice paleo duplications. Nucleic acids research 2009:gkn1048 $\% @ 0305-1048$

68. Ozkan H, Levy AA, Feldman M. Allopolyploidy-induced rapid genome evolution in the wheat (Aegilops-Triticum) group. Plant Cell. 2001;13(8): 1735-47.\%@1532-1298X.

69. Chantret N, Salse J, Sabot F, Rahman S, Bellec A, Laubin B, Dubois I, Dossat C, Sourdille P, Joudrier P. Molecular basis of evolutionary events that shaped the hardness locus in diploid and polyploid wheat species (Triticum and Aegilops). Plant Cell. 2005;17(4):1033-45. \%@ 1532-1298X.

70. Tate JA, Ni Z, Scheen A-C, Koh J, Gilbert CA, Lefkowitz D, Chen ZJ, Soltis PS, Soltis DE. Evolution and expression of homeologous loci in Tragopogon miscellus (Asteraceae), a recent and reciprocally formed allopolyploid. Genetics. 2006;173(3):1599-611. \%@ 0016-6731.

71. Buggs RJA, Chamala S, Wu WEI, Gao LU, May GD, Schnable PS, Soltis DE, Soltis PS, Barbazuk W. Characterization of duplicate gene evolution in the recent natural allopolyploid Tragopogon miscellus by next-generation sequencing and Sequenom iPLEX MassARRAY genotyping. Mol Ecol. 2010; 19(s1):132-46.\%@1365-1294X.

72. Zhang J. Rates of conservative and radical nonsynonymous nucleotide substitutions in mammalian nuclear genes. J Mol Evol. 2000;50(1):56-68. \%@0022-2844.

73. Talamas E, Jackson L, Koeberl M, Jackson T, McElwee JL, Hawes NL, Chang B, Jablonski MM, Sidjanin DJ. Early transposable element insertion in intron 9 of the Hsf4 gene results in autosomal recessive cataracts in lop11 and Idis1 mice. Genomics. 2006;88(1):44--51. \%@ 0888-7543.

74. Shimazaki M, Fujita K, Kobayashi H, Suzuki S. Pink-colored grape berry is the result of short insertion in intron of color regulatory gene. PLOS One. 2011; 6(6):e21308.\%@ 21932-26203.

75. Babenko VN, Rogozin IB, Mekhedov SL, Koonin EV. Prevalence of intron gain over intron loss in the evolution of paralogous gene families. Nucleic Acids Res. 2004;32(12):3724-33.\%@ 0305-1048.

76. Chapman JA, Mascher M, Buluç A, Barry K, Georganas E, Session A, Strnadova V, Jenkins J, Sehgal S, Oliker L. A whole-genome shotgun approach for assembling and anchoring the hexaploid bread wheat genome. Genome Biol. 2015;16(1):26.

77. Ming R, Wai CM. Assembling allopolyploid genomes: no longer formidable. Genome Biol. 2015;16(1):27.\%@1465-6906.

78. Burnquist WL, Sorrelles ME, Tanksley S. Characterization of genetic variability in Saccharum germplasm by means of restriction fragment length polymorphism (RFLP) analysis. 1995. p. 1995

79. Lu YH, D'Hont A, Walker DIT, Rao PS, Feldmann P, Glaszmann JC. Relationships among ancestral species of sugarcane revealed with RFLP using single copy maize nuclear probes. Euphytica. 1994;78(1-2):7-18. $\% @ 0014-2336$

80. Nair NV, Nair S, Sreenivasan TV, Mohan M. Analysis of genetic diversity and phylogeny in Saccharum and related genera using RAPD markers. Genet Resour Crop Ev. 1999;46(1):73-9. \%@ 0925-9864.

81. Daniels J, Roach BT. Taxonomy and evolution. Amsterdam: Sugarcane improvement through breeding Elsevier; 1987. p. 7. 